\title{
SHARP HÖLDER CONTINUITY OF THE LYAPUNOV EXPONENT OF FINITELY DIFFERENTIABLE QUASI-PERIODIC COCYCLES
}

\author{
AO CAI, CLAIRE CHAVAUDRET, JIANGONG YOU, AND QI ZHOU
}

\begin{abstract}
We show that if the base frequency is Diophantine, then the Lyapunov exponent of a $C^{k}$ quasi-periodic $S L(2, \mathbb{R})$ cocycle is $1 / 2$ Hölder continuous in the almost reducible regime, if $k$ is large enough. As a consequence, we show that if the frequency is Diophantine, $k$ is large enough, and the potential is $C^{k}$ small, then the integrated density of states of the corresponding quasi-periodic Schrödinger operator is 1/2-Hölder continuous.
\end{abstract}

\section{Introduction}

Let $(M, \mathcal{B}, \mu)$ be a probability space and $f: M \rightarrow M$ be an invertible measure preserving map, assuming $\mu$ is ergodic. Given a measurable function $A: M \rightarrow S L(2, \mathbb{R})$, the linear cocycle given by $A$ over the base dynamics $f$ is the transformation:

$$
(f, A): M \times \mathbb{R}^{2} \rightarrow M \times \mathbb{R}^{2} ;(\theta, v) \mapsto(f(\theta), A(\theta) \cdot v) .
$$

The iterates of $(f, A)$ have the form $(f, A)^{n}=\left(f^{n}, A_{n}\right)$, where $A_{n}(\theta)=$ $A\left(f^{n-1}(\theta)\right) \cdots A(f(\theta)) A(\theta), n \geqslant 1$ and $A_{-n}(\theta)=A_{n}\left(f^{-n}(\theta)\right)^{-1}$. The Lyapunov exponent is given by the formula

$$
L(f, A)=\lim _{n \rightarrow+\infty} \frac{1}{n} \int_{M} \ln \left\|A_{n}(\theta)\right\| d \mu .
$$

Lyapunov exponents appear naturally in the study of smooth dynamics. Continuity of Lyapunov exponents depends sensitively on the finer topologies of the fiber and the base dynamics. This has been the object of considerable recent interests, see Viana [31] and references therein. It is well known that in $C^{0}$ topology, discontinuity of Lyapunov exponent occurs at every non-uniformly hyperbolic cocycle, see [21, 28, 30]. Moreover, Bochi $[12,13]$ proved that with an ergodic base system, any non-uniformly hyperbolic $S L(2, \mathbb{R})$-cocycle can be approximated by cocycles with zero Lyapunov exponents in the $C^{0}$ topology. Therefore, if one wants to obtain some regularity of the Lyapunov exponent, finer topology than $C^{0}$ topology is necessary.

Now we discuss the impact of the base dynamics. If the base dynamics has some hyperbolicity, then the Lyapunov exponent is continuous. For example, Bocker and Viana [11] proved continuity of Lyapunov exponents for 
random products of $S L(2, \mathbb{R})$ matrices in the Bernoulli setting. In higher dimensions, continuous dependence of all Lyapunov exponents for i.i.d. random products of matrices in $G L(d, R)$ was proved by Avila, Eskin, and Viana [7]. If the base dynamics is a subshift of finite type or, more generally, a hyperbolic set, then Backes, Brown, Butler [9] proved that Lyapunov exponents are always continuous among Hölder continuous fiber-bunched $S L(2, \mathbb{R})$-cocycles, which answers a famous conjecture of Viana [31].

If the base is elliptic, things become more complicated: it will depend on the smoothness of $A(\theta)$ in a very sensitive way. Now assume that the base dynamics is quasiperiodic, i.e. $f: \mathbb{T}^{d} \rightarrow \mathbb{T}^{d}$ with $f(\theta)=\theta+\alpha$, where $\alpha$ is rationally independent. If $A(\theta)$ is analytic, the Lyapunov exponent is always continuous $[14,15,26]$, even in high dimensional $G L(d, \mathbb{C})$ cocycles (see [5]). However, if $A(\theta)$ is finitely differentiable, Wang and You [32] constructed examples of discontinuity of the Lyapunov exponent in smooth quasi-periodic $S L(2, \mathbb{R})$ cocycles even when $\alpha$ is of bounded type.

In this paper, we will show that if $A \in C^{k}\left(\mathbb{T}^{d}, S L(2, \mathbb{R})\right)$, Lyapunov exponent can still be continuous, even Hölder continuous provided that the cocycle is almost reducible. Before explaining our result precisely, we introduce some basic concepts. Recall that $\alpha \in \mathbb{R}^{d}$ is called Diophantine if there are $\kappa>0$ and $\tau>d-1$ such that $\alpha \in \mathrm{DC}(\kappa, \tau)$, where

$$
\operatorname{DC}(\kappa, \tau):=\left\{\omega \in \mathbb{R}^{d}: \inf _{j \in \mathbb{Z}}|\langle n, \omega\rangle-j|>\frac{\kappa}{|n| \tau}, \quad \forall n \in \mathbb{Z}^{d} \backslash\{0\}\right\} .
$$

We also set DC $:=\bigcup_{\kappa>0, \tau>d-1} \mathrm{DC}(\kappa, \tau)$. Given two cocycles $\left(\alpha, A_{1}\right),\left(\alpha, A_{2}\right) \in$ $\mathbb{T}^{d} \times C^{k^{\prime}}\left(\mathbb{T}^{d}, S L(2, \mathbb{R})\right)$, one says that they are $C^{k}$ conjugated if there exists $Z \in C^{k}\left(2 \mathbb{T}^{d}, S L(2, \mathbb{R})\right)$, such that

$$
Z(\theta+\alpha)^{-1} A_{1}(\theta) Z(\theta)=A_{2}(\theta) .
$$

We say $(\alpha, A)$ is $C^{k^{\prime}, k}$ almost reducible, if $A \in C^{k^{\prime}}\left(\mathbb{T}^{d}, S L(2, \mathbb{R})\right)$, and closure of its $C^{k}$ conjugacies contains a constant. Within the above concepts, our main result is the following:

Theorem 1.1. Let $\alpha \in D C(\kappa, \tau)$, there exists a numerical constant $D_{0}>0$, such that if $(\alpha, A)$ is $C^{k^{\prime}, k}$ almost reducible with $k^{\prime}>k \geqslant D_{0} \tau$, then for any continuous map $B: \mathbb{T}^{d} \rightarrow S L(2, \mathbb{C})$, we have

$$
|L(\alpha, A)-L(\alpha, B)| \leqslant C_{0}\|B-A\|_{0}^{\frac{1}{2}},
$$

where $C_{0}$ is a constant depending on $d, \kappa, \tau$.

Typical example of $S L(2, \mathbb{R})$ cocycles comes from the Schrödinger cocycles, where

$$
A(\theta)=S_{E}^{\lambda V}=\left(\begin{array}{cc}
\lambda V(\theta)-E & -1 \\
1 & 0
\end{array}\right) .
$$

They come from one dimensional quasi-periodic Schrödinger operators on $\ell^{2}(\mathbb{Z})$ :

$$
\left(H_{\lambda V, \alpha, \theta} u\right)_{n}=u_{n+1}+u_{n-1}+\lambda V(\theta+n \alpha) u_{n}=E u_{n}
$$


since a solution $H_{\lambda V, \alpha, \theta} u=E u$ satisfies $A(\theta+n \alpha)\left(\begin{array}{c}u_{n} \\ u_{n-1}\end{array}\right)=\left(\begin{array}{c}u_{n+1} \\ u_{n}\end{array}\right)$.

For Schrödinger operators $H_{\lambda V, \alpha, \theta}$, another important concept related to the Lyapunov exponent is the integrated density of states (IDS), which is the function $N: \mathbb{R} \rightarrow[0,1]$ defined by

$$
N_{\lambda V, \alpha}(E)=\int_{\mathbb{T}^{d}} \mu_{\lambda V, \alpha, \theta}(-\infty, E] d \theta,
$$

where $\mu_{\lambda V, \alpha, \theta}$ is the spectral measure of $H_{\lambda V, \alpha, \theta}$. As a local version of our main result, we are able to show the following:

Theorem 1.2. Let $\alpha \in D C(\kappa, \tau), V \in C^{k^{\prime}}\left(\mathbb{T}^{d}, \mathbb{R}\right)$ with $k^{\prime} \geqslant D_{0} \tau$, where $D_{0}$ is a numerical constant. Then there exist $\lambda_{0}, k$ depending on $V, d, \kappa, \tau, k^{\prime}$ such that if $\lambda<\lambda_{0}$, then we have the following:

(1) For any $E \in \mathbb{R},\left(\alpha, S_{E}^{\lambda V}\right)$ is $C^{k^{\prime}, k}$ almost reducible.

(2) $N_{\lambda V, \alpha}$ is $1 / 2$-Hölder continuous.

Remark 1.1. We provide the first positive result of Hölder continuity of the Lyapunov exponent for finitely differentiable $S L(2, \mathbb{R})$ cocycles. This kind of $1 / 2$-Hölder continuity is sharp due to the presence of spectral gaps.

Remark 1.2. Our choice of $k^{\prime}$ and $k$ is not optimal, readers are invited to consult section 3.5 for more discussions.

Before giving the rough ideas of the proof, let us first go over more history on the study of high order regularity of Lyapunov exponents. Just note that by the Thouless formula, (weak) Hölder continuity of the Lyapunov exponent is equivalent to (weak) Hölder continuity of the integrated density of states.

If the cocycle is analytic, one should distinguish zero Lyapunov exponent and positive Lyapunov exponent. In the positive Lyapunov exponent regime, it starts with the work of Goldstein and Schlag [22] where they developed some sharp version of large deviation theorems for real analytic potentials with strong Diophantine frequency; they further developed Avalanche Principle and proved that Lyapunov exponent is Hölder continuous (onefrequency) or weak Hölder continuous (multi-frequency). For the Almost Mathieu operator $H_{\lambda}=\lambda \cos 2 \pi(k \omega+\theta)+\triangle$, Bourgain [16] proved that for large $\lambda$ depending on Diophantine $\omega$, the Lyapunov exponent is $\frac{1}{2}-\epsilon$ Hölder continuous for any $\epsilon>0$. Later, Goldstein and Schlag [23] generalized Bourgain's result [16], and proved that if the potential is in a small $L^{\infty}$ neighborhood of a trigonometric polynomial of degree $k$, then the IDS is Hölder $\frac{1}{2 k}-\epsilon$-continuous for all $\epsilon>0$.

On the other hand, in the zero Lyapunov exponent regime, based on Eliasson's perturbative KAM scheme [20], Amor [1] obtained 1/2-Hölder continuity of the IDS for quasi-periodic Schrödinger operator with a Diophantine frequency and small potential. Avila and Jitomirskaya [4] further proved that if the cocycle is $C^{\omega}$ almost reducible (consult section 2.1 for definitions), then the Lyapunov exponent is $1 / 2$ Hölder continous. Thus our 
result generalized Avila and Jitomirskaya's result [4] to the differentiable case.

Notice that all the results stated above require Diophantine or strong Diophantine conditions. In fact, for small potential and generic frequencies, it is possible to show that the Lyapunov exponent is not Hölder continuous [3]. A recent breakthrough belongs to Avila [3]: for one-frequency Schrödinger operators with general analytic potentials and irrational frequency, Avila has built the fantastic global theory saying that the Lyapunov exponent is a $C^{\omega}$-stratified function of the energy.

In the lower regularity case, Klein [27] proved that for Schrödinger operators with potentials in a Gevrey class, the Lyapunov exponent is weak Hölder continuous on any compact interval of the energy provided that the coupling constant is large enough, the frequency is Diophantine and the potential satisfies some transversality condition. Recently, Wang and Zhang [33] obtained the weak Hölder continuity of the Lyapunov exponent as function of energies, for a class of $C^{2}$ quasi-periodic potentials and for any Diophantine frequency.

For other related results, Avila and Krikorian [6] have studied so-called monotonic cocycles which are a class of smooth or analytic cocycles nonhomotopic to constant. They showed that the Lyapunov exponent is smooth or even analytic, respectively. Besides, for a 1-periodic function satisfying a Lipschitz monotonicity condition, Jitomirskaya and Kachkovskiy [25] showed that the Lyapunov exponent is almost Lipschitz continuous.

Finally, we will comment on the method of the proof. Our method is purely dynamical, and the philosophy is that nice quantitative almost reducibility implies nice spectral applications. This approach, which was first developed in [20], has been proved to be very fruitful $[2,4,8,29]$. To our purposes, the key is to have a nice control of the growth of the cocycles, i.e. to have a nice control of the $C^{0}$ norm of the conjugacy and the perturbation in the almost reducible scheme. Since our system is $C^{k}$ instead of analytic, we will first perform the KAM scheme in the analytic topology, then obtain the corresponding $C^{k}$ estimates by analytic approximations [34], which is very classical in KAM theory. In order to do the analytic approximation, we need the strong almost reducibility results (i.e. the cocycle is almost reducible in a fixed band, and this band is arbitrarily close to the initial band). Although this kind of result was first obtained by Chavaudret [17], however, her estimates there are too rough to have good spectral applications (readers can consult section 3.5 for more discussions). Instead, we will perform a new KAM scheme which was developed by Leguil-You-Zhao-Zhou [29], where they initially developed the scheme to obtain the exponential asymptotics on the size of spectral gaps for almost Mathieu operators. With the KAM scheme [29] in hand, the key is to choose the suitable analytic approximation, to have sufficiently subtle quantitative estimates. 


\section{Preliminaries}

For a bounded analytic (possibly matrix-valued) function $F$ defined on $\{\theta|| \Im \theta \mid<h\}$, let $\|F\|_{h}=\sup _{|\Im \theta|<h}\|F(\theta)\|$ and denote by $C_{h}^{\omega}\left(\mathbb{T}^{d}, *\right)$ the set of all these $*$-valued functions ( $*$ will usually denote $\mathbb{R}, \operatorname{sl}(2, \mathbb{R}) S L(2, \mathbb{R})$ ). Also we denote $C^{\omega}\left(\mathbb{T}^{d}, *\right)=\cup_{h>0} C_{h}^{\omega}\left(\mathbb{T}^{d}, *\right)$, and set $C^{k}\left(\mathbb{T}^{d}, *\right)$ to be the space of $k$ times differentiable matrix-valued functions. The norms are defined as

$$
\|F\|_{k}=\sup _{k^{\prime} \leqslant k, \theta \in \mathbb{T}^{d}}\left\|\partial^{k^{\prime}} F(\theta)\right\|
$$

and

$$
\|F\|_{0}=\sup _{\theta \in \mathbb{T}^{d}}\|F(\theta)\|
$$

For any $N>0$, define the truncating operators $\mathcal{T}_{N}$ on $C^{\omega}\left(\mathbb{T}^{d}, *\right)$ as

$$
\left(\mathcal{T}_{N} f\right)(\theta)=\sum_{k \in \mathbb{Z}^{d},|k|<N} \hat{f}(k) e^{i<k, \theta>}
$$

and $\mathcal{R}_{N}$ as

$$
\left(\mathcal{R}_{N} f\right)(\theta)=\sum_{k \in \mathbb{Z}^{d},|k| \geqslant N} \hat{f}(k) e^{i<k, \theta>} .
$$

2.1. Hyperbolicity and (almost) reducibility. We say the cocycle $(\alpha, A)$ is uniformly hyperbolic if for every $\theta \in \mathbb{T}^{d}$, there exists a continuous splitting $\mathbb{C}^{2}=E^{s}(\theta) \oplus E^{u}(\theta)$ such that for some constants $C>0, c>0$, and for every $n \geqslant 0$,

$$
\begin{aligned}
\left|A_{n}(\theta) v\right| & \leqslant C e^{-c n}|v|, v \in E^{s}(\theta), \\
\left|A_{n}(\theta)^{-1} v\right| & \leqslant C e^{-c n}|v|, v \in E^{u}(\theta+n \alpha) .
\end{aligned}
$$

This splitting is invariant by the dynamics, which means that for every $\theta \in \mathbb{T}^{d}, A(\theta) E^{*}(\theta)=E^{*}(\theta+\alpha)$, for $*=s, u$. In this case, it is clear that we have $L(\alpha, A)>0$.

Given two analytic cocycles $\left(\alpha, A_{1}\right),\left(\alpha, A_{2}\right) \in \mathbb{T}^{d} \times C^{\omega}\left(\mathbb{T}^{d}, S L(2, \mathbb{R})\right)$, they are analytically conjugated if there exists $Z \in C^{\omega}\left(2 \mathbb{T}^{d}, S L(2, \mathbb{R})\right)$, such that

$$
Z(\theta+\alpha)^{-1} A_{1}(\theta) Z(\theta)=A_{2}(\theta) .
$$

We call the cocycle $(\alpha, A)$ is $C^{\omega}$ almost reducible if the closure of its analytic conjugacy class contains a constant.

2.2. Analytic approximation. Assume $f \in C^{k}\left(\mathbb{T}^{d}, s l(2, \mathbb{R})\right)$. By [34], there exists a sequence $\left(f_{j}\right)_{j \geqslant 1}, f_{j} \in C_{\frac{1}{j}}^{\omega}\left(\mathbb{T}^{d}, \operatorname{sl}(2, \mathbb{R})\right)$ and a universal constant $C^{\prime}$, such that

$$
\begin{aligned}
\left\|f_{j}-f\right\|_{k} & \rightarrow 0, \quad j \rightarrow+\infty, \\
\left|f_{j}\right|_{\frac{1}{j}} & \leqslant C^{\prime}\|f\|_{k}, \\
\left|f_{j+1}-f_{j}\right|_{\frac{1}{j+1}} & \leqslant C^{\prime}\left(\frac{1}{j}\right)^{k}\|f\|_{k} .
\end{aligned}
$$


Moreover, if $k \leqslant k^{\prime}$ and $f \in C^{k^{\prime}}$, then properties (2.1) hold with $k^{\prime}$ instead of $k$. That means this sequence is obtained from $f$ regardless of its regularity (since $f_{j}$ is the convolution of $F$ with a map which does not depend on $k$ ).

\section{Quantitative estimates of almost Reducibility}

The concept of $C^{k^{\prime}, k}$ almost reducibility is merely qualitative and does not involve any quantitative estimates. However, the surprising thing is that one can really derive quite good quantitative estimates, since this concept captures the essential of applicability of local theories. In order to give almost reducibility theorem for $C^{k}$ quasi-periodic $S L(2, \mathbb{R})$ cocycle, we are not going to perform a KAM scheme straightly on a $C^{k}$ cocycle. Instead, we will deal with almost reducibility for analytic cocycles and turn it into a finitely differentiable analogue by virtue of analytic approximation. So we first treat the analytic case before dealing with the differentiable case.

3.1. Decomposition along resonances. In the following subsection, parameters $\rho, \epsilon, N, \sigma$ will be fixed; one will refer to the situation where there exists $n_{*}$ with $0<\left|n_{*}\right| \leqslant N$ such that

$$
\left|2 \rho-<n_{*}, \alpha>\right|<\epsilon^{\sigma},
$$

as the "resonant case". The integer vector $n_{*}$ will be referred to as a "resonant site".

Resonances are linked to a useful decomposition of the space $C_{r}^{\omega}\left(\mathbb{T}^{d}, s u(1,1)\right)$ which is defined as follows: for any given $\eta>0, \alpha \in \mathbb{R}^{d}$ and $A \in S U(1,1)$, we decompose $\mathcal{B}_{r}=C_{r}^{\omega}\left(\mathbb{T}^{d}, s u(1,1)\right)=\mathcal{B}_{r}^{\text {nre }}(\eta) \bigoplus \mathcal{B}_{r}^{r e}(\eta)$ in such a way that for any $Y \in \mathcal{B}_{r}^{\text {nre }}(\eta)$,

$$
A^{-1} Y(\theta+\alpha) A \in \mathcal{B}_{r}^{n r e}(\eta), \quad\left|A^{-1} Y(\theta+\alpha) A-Y(\theta)\right|_{r} \geqslant \eta|Y(\theta)|_{r}
$$

Moreover, let $\mathbb{P}_{\text {nre }}$ and $\mathbb{P}_{r e}$ be the standard projections from $\mathcal{B}_{r}$ onto $\mathcal{B}_{r}^{\text {nre }}(\eta)$ and $\mathcal{B}_{r}^{r e}(\eta)$ respectively.

Within the above notations, the basic fact for our purpose is the following result:

Lemma 3.1. Assume that $\epsilon \leqslant(4\|A\|)^{-4}$ and $\eta \geqslant 13\|A\|^{2} \epsilon^{\frac{1}{2}}$. For any $g \in \mathcal{B}_{r}$ with $|g|_{r} \leqslant \epsilon$, there exist $Y \in \mathcal{B}_{r}$ and $g^{r e} \in \mathcal{B}_{r}^{r e}(\eta)$ such that

$$
e^{Y(\theta+\alpha)}\left(A e^{g(\theta)}\right) e^{-Y(\theta)}=A e^{g^{r e}(\theta)},
$$

with $|Y|_{r} \leqslant \epsilon^{\frac{1}{2}}$ and $\left|g^{r e}\right|_{r} \leqslant 2 \epsilon$.

Remark 3.1. The continuous version of this result appears in Lemma 3.1 of [24]. We will give a different proof using the quantitative Implicit Function Theorem in the Appendix. Just point out that the proof only relies on the fact that $\mathcal{B}_{r}$ is a Banach space, thus it also applies to $C^{k}$ and $C^{0}$ topology. 
3.2. Analytic case. In this subsection, we concentrate on the following analytic quasi-periodic $S L(2, \mathbb{R})$ cocycle:

$$
\left(\alpha, A e^{f(\theta)}\right): \mathbb{T}^{d} \times \mathbb{R}^{2} \rightarrow \mathbb{T}^{d} \times \mathbb{R}^{2} ;(\theta, v) \mapsto\left(\theta+\alpha, A e^{f(\theta)} \cdot v\right),
$$

where $f \in C_{r_{0}}^{\omega}\left(\mathbb{T}^{d}, s l(2, \mathbb{R})\right), \quad r_{0}>0, d \in \mathbb{N}^{+}$and $\alpha \in D C(\kappa, \tau)$. Notice that $A$ has eigenvalues $\left\{e^{i \rho}, e^{-i \rho}\right\}$ with $\rho \in \mathbb{R} \cup i \mathbb{R}$.

Through the proof, we find that analytic approximation requires us to show a result concerning a stronger sense of almost reducibility, i.e. we may shrink the analytic radius as little as we want in each iteration step and the perturbation still tends to zero. Moreover, we must have a good control of the analytic norm of the conjugation map.

Proposition 3.1. Let $\alpha \in D C(\kappa, \tau), \kappa, r>0, \tau>d-1, \sigma=\frac{1}{10}$. Suppose that $A \in S L(2, \mathbb{R}), f \in C_{r}^{\omega}\left(\mathbb{T}^{d}, s l(2, \mathbb{R})\right)$. Then for any $r^{\prime} \in(0, r)$, there exist $c=c(\kappa, \tau, d)$ and a numerical constant $D$ such that if

$$
|f|_{r} \leqslant \epsilon \leqslant \frac{c}{\|A\|^{D}}\left(r-r^{\prime}\right)^{D \tau}
$$

then there exist $B \in C_{r^{\prime}}^{\omega}\left(2 \mathbb{T}^{d}, S L(2, \mathbb{R})\right), A_{+} \in S L(2, \mathbb{R})$ and $f_{+} \in C_{r^{\prime}}^{\omega}\left(\mathbb{T}^{d}\right.$, sl $(2, \mathbb{R}))$ such that

$$
B(\theta+\alpha)\left(A e^{f(\theta)}\right) B^{-1}(\theta)=A_{+} e^{f_{+}(\theta)} .
$$

More precisely, let $N=\frac{2}{r-r^{\prime}}|\ln \epsilon|$, then we can distinguish two cases:

- (Non-resonant case) if for any $n \in \mathbb{Z}^{d}$ with $0<|n| \leqslant N$, we have

$$
|2 \rho-<n, \alpha>| \geqslant \epsilon^{\sigma},
$$

then

$$
|B-I d|_{r^{\prime}} \leqslant \epsilon^{\frac{1}{2}}, \quad\left|f_{+}\right|_{r^{\prime}} \leqslant 4 \epsilon^{3-2 \sigma} .
$$

and

$$
\left\|A_{+}-A\right\| \leqslant 2\|A\| \epsilon
$$

- (Resonant case) if there exists $n_{*}$ with $0<\left|n_{*}\right| \leqslant N$ such that

$$
\left|2 \rho-<n_{*}, \alpha>\right|<\epsilon^{\sigma},
$$

then

$$
\begin{aligned}
& |B|_{r^{\prime}} \leqslant \epsilon^{-\frac{\sigma}{10}} \times \epsilon^{\frac{-r^{\prime}}{r-r^{\prime}}}, \quad\|B\|_{0} \leqslant \epsilon^{-\frac{\sigma}{10}}, \quad\left|f_{+}\right|_{r^{\prime}} \ll \epsilon^{100} . \\
& \text { Moreover, } A_{+}=e^{A^{\prime \prime}} \text { with }\left\|A^{\prime \prime}\right\| \leqslant 2 \epsilon^{\sigma} .
\end{aligned}
$$

Proof. Although the result was stated for the $S L(2, \mathbb{R})$ case, we prefer to prove it in the isomorphic group $S U(1,1)$, since the proof will become more explicit. Notice that $s l(2, \mathbb{R})$ is isomorphic to $s u(1,1)$, which consists of matrices of the form

$$
\left(\begin{array}{cc}
i t & v \\
\bar{v} & -i t
\end{array}\right)
$$


with $t \in \mathbb{R}, v \in \mathbb{C}$. The isomorphism between them is given by $A \rightarrow$ $M A M^{-1}$, where

$$
M=\frac{1}{1+i}\left(\begin{array}{cc}
1 & -i \\
1 & i
\end{array}\right)
$$

and a simple calculation yields

$$
M\left(\begin{array}{cc}
x & y+z \\
y-z & -x
\end{array}\right) M^{-1}=\left(\begin{array}{cc}
i z & x-i y \\
x+i y & -i z
\end{array}\right)
$$

Now we divide the proof into two cases:

Non-resonant case: For $0<|n| \leqslant N=\frac{2}{r-r^{\prime}}|\ln \epsilon|$, we have

$$
|2 \rho-<n, \alpha>| \geqslant \epsilon^{\sigma}
$$

by (3.2) with $D \geqslant 110$, we have

$$
|<n, \alpha>| \geqslant \frac{\kappa}{|n|^{\tau}} \geqslant \frac{\kappa}{|N|^{\tau}} \geqslant \epsilon^{\sigma} .
$$

Define

$$
\Lambda_{N}=\left\{f \in C_{r}^{\omega}\left(\mathbb{T}^{d}, s u(1,1)\right) \mid f(\theta)=\sum_{k \in \mathbb{Z}^{d}, 0<|k|<N} \hat{f}(k) e^{i<k, \theta>}\right\} .
$$

By (3.3) and (3.4), direct computation shows that if $Y \in \Lambda_{N}$, then

$$
\left|A^{-1} Y(\theta+\alpha) A-Y(\theta)\right|_{r} \geqslant \epsilon^{3 \sigma}|Y(\theta)|_{r},
$$

thus $\Lambda_{N} \subset \mathcal{B}_{r}^{\text {nre }}\left(\epsilon^{3 \sigma}\right)$. Since $\epsilon^{3 \sigma} \geqslant 13\|A\|^{2} \epsilon^{\frac{1}{2}}$, by Lemma 3.1 we have $Y \in \mathcal{B}_{r}$ and $f^{r e} \in \mathcal{B}_{r}^{r e}\left(\epsilon^{3 \sigma}\right)$ such that

$$
e^{Y(\theta+\alpha)}\left(A e^{f(\theta)}\right) e^{-Y(\theta)}=A e^{f^{r e}(\theta)},
$$

with $|Y|_{r} \leqslant \epsilon^{\frac{1}{2}}$ and

$$
\left|f^{r e}\right|_{r} \leqslant 2 \epsilon
$$

By (3.5)

$$
\left(\mathcal{T}_{N} f^{r e}\right)(\theta)=\hat{f}^{r e}(0), \quad\left\|\hat{f}^{r e}(0)\right\| \leqslant 2 \epsilon
$$

and

$$
\begin{aligned}
\left|\left(\mathcal{R}_{N} f^{r e}\right)(\theta)\right|_{r^{\prime}} & =\left|\sum_{|n|>N} \hat{f}^{r e}(n) e^{i<n, \theta>}\right|_{r^{\prime}} \\
& \leqslant 2 \epsilon e^{-N\left(r-r^{\prime}\right)}(N)^{d} \\
& \leqslant 2 \epsilon^{3-2 \sigma} .
\end{aligned}
$$

Moreover, we can compute that

$$
e^{\hat{f}^{r e}(0)+\mathcal{R}_{N} f^{r e}(\theta)}=e^{\hat{f}^{r e}(0)}\left(I d+e^{-\hat{f}^{r e}(0)} \mathcal{O}\left(\mathcal{R}_{N} f^{r e}\right)\right)=e^{\hat{f}^{r e}(0)} e^{f_{+}(\theta)},
$$

by (3.7), we have

$$
\left|f_{+}(\theta)\right|_{r^{\prime}} \leqslant 2\left|\mathcal{R}_{N} f^{r e}(\theta)\right|_{r^{\prime}} \leqslant 4 \epsilon^{3-2 \sigma} .
$$


Finally, if we denote

$$
A_{+}=A e^{\hat{f}^{r e}(0)}
$$

then we have

$$
\left\|A_{+}-A\right\| \leqslant\|A\|\left\|I d-e^{\hat{f}^{r e}(0)}\right\| \leqslant 2\|A\| \epsilon .
$$

Resonant case: Note that we only need to consider the case in which $A$ is elliptic with eigenvalues $\left\{e^{i \rho}, e^{-i \rho}\right\}$ for $\rho \in \mathbb{R} \backslash\{0\}$, because if $\rho \in i \mathbb{R}$, the non-resonant condition is always fulfilled due to the Diophantine condition on $\alpha$ and then it indeed belongs to the non-resonant case.

Claim: $n_{*}$ is the unique resonant site with

$$
0<\left|n_{*}\right| \leqslant N=\frac{2}{r-r^{\prime}}|\ln \epsilon| .
$$

Proof. Indeed, if there exists $n_{*}^{\prime} \neq n_{*}$ satisfying $\left|2 \rho-<n_{*}^{\prime}, \alpha>\right|<\epsilon^{\sigma}$, then by the Diophantine condition of $\alpha$, we have

$$
\frac{\kappa}{\left|n_{*}^{\prime}-n_{*}\right| \tau} \leqslant\left|<n_{*}^{\prime}-n_{*}, \alpha>\right|<2 \epsilon^{\sigma},
$$

which implies that $\left|n_{*}^{\prime}\right|>2^{-\frac{1}{\tau}} \kappa^{\frac{1}{\tau}} \epsilon^{-\frac{\sigma}{\tau}}-N \gg N$.

Since we have

$$
\left|2 \rho-<n_{*}, \alpha>\right|<\epsilon^{\sigma},
$$

the smallness condition on $\epsilon$ implies that

$$
|\ln \epsilon|^{\tau} \epsilon^{\sigma} \leqslant \frac{\kappa\left(r-r^{\prime}\right)^{\tau}}{2^{\tau+1}}
$$

Thus

$$
\frac{\kappa}{\left|n_{*}\right|^{\tau}} \leqslant\left|<n_{*}, \alpha>\right| \leqslant \epsilon^{\sigma}+2|\rho| \leqslant \frac{\kappa}{2\left|n_{*}\right|^{\tau}}+2|\rho|,
$$

which implies that

$$
|\rho| \geqslant \frac{\kappa}{4\left|n_{*}\right| \tau} .
$$

Thus one can find $P \in S U(1,1)$ with

$$
\|P\| \leqslant \frac{2\|A\|}{|\rho|} \leqslant \frac{8\|A\|}{\kappa}\left|n_{*}\right|^{\tau},
$$

such that

$$
P A P^{-1}=\left(\begin{array}{cc}
e^{i \rho} & 0 \\
0 & e^{-i \rho}
\end{array}\right)=A^{\prime} .
$$

Denote $g=\operatorname{Pf} P^{-1}$, by (3.2) with $D \geqslant 110$, we have

$$
\|A\|\left|n_{*}\right|^{\tau} \leqslant\|A\| N^{\tau} \leqslant \epsilon^{-\frac{\sigma}{11}},
$$


thus one gets the following estimates:

$$
\begin{aligned}
\|P\| & \leqslant \frac{8\|A\|}{\kappa} N^{\tau} \leqslant \frac{1}{2} \epsilon^{-\frac{\sigma}{10}}, \\
|g|_{r} & \leqslant\|P\|^{2}|f|_{r} \leqslant \frac{1}{4} \epsilon^{1-\frac{\sigma}{5}}:=\epsilon^{\prime} .
\end{aligned}
$$

Now we define

$$
\begin{aligned}
& \Lambda_{1}\left(\epsilon^{\sigma}\right)=\left\{n \in \mathbb{Z}^{d}:|<n, \alpha>| \geqslant \epsilon^{\sigma}\right\}, \\
& \Lambda_{2}\left(\epsilon^{\sigma}\right)=\left\{n \in \mathbb{Z}^{d}:|2 \rho-<n, \alpha>| \geqslant \epsilon^{\sigma}\right\},
\end{aligned}
$$

and define $\mathcal{B}_{r}^{\text {nre }}\left(\epsilon^{\sigma}\right)$ as in (3.1) with $A$ substituted by $A^{\prime}$, then we can compute that any $Y \in \mathcal{B}_{r}^{\text {nre }}\left(\epsilon^{\sigma}\right)$ takes the precise form:

$$
\begin{aligned}
Y(\theta)= & \sum_{n \in \Lambda_{1}\left(\epsilon^{\sigma}\right)}\left(\begin{array}{cc}
i \hat{t}(n) & 0 \\
0 & -i \hat{t}(n)
\end{array}\right) e^{i<n, \theta>}+ \\
& \sum_{n \in \Lambda_{2}\left(\epsilon^{\sigma}\right)}\left(\begin{array}{cc}
0 & \hat{v}(n) e^{i<n, \theta>} \\
\hat{v}(n) e^{-i<n, \theta>} & 0
\end{array}\right) .
\end{aligned}
$$

Since $\epsilon^{\sigma} \geqslant 13\left\|A^{\prime}\right\|^{2}\left(\epsilon^{\prime}\right)^{\frac{1}{2}}$, we can apply Lemma 3.1 to remove all the nonresonant terms of $g$, which means there exist $Y \in \mathcal{B}_{r}$ and $g^{r e} \in \mathcal{B}_{r}^{r e}(\eta)$ such that

$$
e^{Y(\theta+\alpha)}\left(A^{\prime} e^{g(\theta)}\right) e^{-Y(\theta)}=A^{\prime} e^{g^{r e}(\theta)},
$$

with $|Y|_{r} \leqslant\left(\epsilon^{\prime}\right)^{\frac{1}{2}}$ and $\left|g^{r e}\right|_{r} \leqslant 2 \epsilon^{\prime}$.

Combining with the Diophantine condition on the frequency $\alpha$ and the Claim, we have:

$$
\begin{aligned}
& \left\{\mathbb{Z}^{d} \backslash \Lambda_{1}\left(\epsilon^{\sigma}\right)\right\} \cap\left\{n \in \mathbb{Z}^{d}:|n| \leqslant \kappa^{\frac{1}{\tau}} \epsilon^{-\frac{\sigma}{\tau}}\right\}=\{0\}, \\
& \left\{\mathbb{Z}^{d} \backslash \Lambda_{2}\left(\epsilon^{\sigma}\right)\right\} \cap\left\{n \in \mathbb{Z}^{d}:|n| \leqslant 2^{-\frac{1}{\tau}} \kappa^{\frac{1}{\tau}} \epsilon^{-\frac{\sigma}{\tau}}-N\right\}=\left\{n_{*}\right\} .
\end{aligned}
$$

Let $N^{\prime}:=2^{-\frac{1}{\tau}} \kappa^{\frac{1}{\tau}} \epsilon^{-\frac{\sigma}{\tau}}-N$, then we can rewrite $g^{r e}(\theta)$ as

$$
\begin{aligned}
g^{r e}(\theta) & =g_{0}^{r e}+g_{1}^{r e}(\theta)+g_{2}^{r e}(\theta) \\
& =\left(\begin{array}{cc}
i \hat{t}(0) & 0 \\
0 & -i \hat{t}(0)
\end{array}\right)+\left(\begin{array}{cc}
0 & \hat{v}\left(n_{*}\right) e^{i<n_{*}, \theta>} \\
& +\sum_{|n|>N^{\prime}} \hat{g}^{r e}(n) e^{i<n, \theta>}
\end{array}\right)
\end{aligned}
$$

Define the $4 \pi \mathbb{Z}$-periodic rotation $Q(\theta)$ as below:

$$
Q(\theta)=\left(\begin{array}{cc}
e^{-\frac{\left\langle n_{*}, \theta\right\rangle_{i}}{2} i} & 0 \\
0 & e^{\frac{\leq n_{*}, \theta>_{i}}{2} i}
\end{array}\right) .
$$

So we have

$$
|Q(\theta)|_{r^{\prime}} \leqslant e^{\frac{1}{2} N r^{\prime}} \leqslant \epsilon^{\frac{-r^{\prime}}{r-r^{\prime}}}
$$


One can also show that

$$
Q(\theta+\alpha)\left(A^{\prime} e^{g^{r e}(\theta)}\right) Q^{-1}(\theta)=\tilde{A} e^{\tilde{g}(\theta)},
$$

where

$$
\tilde{A}=Q(\theta+\alpha) A^{\prime} Q^{-1}(\theta)=\left(\begin{array}{cc}
e^{i\left(\rho-\frac{<n_{*}, \omega>}{2}\right)} & 0 \\
0 & e^{-i\left(\rho-\frac{<n_{*}, \omega>}{2}\right)}
\end{array}\right)
$$

and

$$
\tilde{g}(\theta)=Q g^{r e}(\theta) Q^{-1}=Q g_{0}^{r e} Q^{-1}+Q g_{1}^{r e}(\theta) Q^{-1}+Q g_{2}^{r e}(\theta) Q^{-1} .
$$

Moreover,

$$
\begin{aligned}
Q g_{0}^{r e} Q^{-1} & =g_{0}^{r e}=\left(\begin{array}{cc}
i \hat{t}(0) & 0 \\
0 & -i \hat{t}(0)
\end{array}\right) \in s u(1,1), \\
Q g_{1}^{r e}(\theta) Q^{-1} & =\left(\begin{array}{cc}
0 & \hat{v}\left(n_{*}\right) \\
\hat{v}\left(n_{*}\right) & 0
\end{array}\right) \in s u(1,1),
\end{aligned}
$$

Now we return back from $s u(1,1)$ to $s l(2, \mathbb{R})$. Denote

$$
\begin{aligned}
L & =M^{-1}\left(Q g_{0}^{r e} Q^{-1}+Q g_{1}^{r e}(\theta) Q^{-1}\right) M, \\
F & =M^{-1} Q g_{2}^{r e}(\theta) Q^{-1} M, \\
B & =M^{-1}\left(Q \circ e^{Y} \circ P\right) M, \\
\tilde{A}^{\prime} & =M^{-1} \tilde{A} M,
\end{aligned}
$$

then we have:

$$
B(\theta+\alpha)\left(A e^{f(\theta)}\right) B^{-1}(\theta)=\tilde{A}^{\prime} e^{L+F(\theta)},
$$

by (3.9) and (3.12), we have the following estimates:

(3.14) $\|B\|_{0} \leqslant\left|e^{Y}\right|_{r}\|P\| \leqslant \epsilon^{-\frac{\sigma}{10}}$,

$(3.15)|B|_{r^{\prime}} \leqslant \epsilon^{-\frac{\sigma}{10}} \times \epsilon^{\frac{-r^{\prime}}{r-r^{\prime}}}$,

(3.16) $\|L\| \leqslant\left\|Q g_{0}^{r e} Q^{-1}\right\|+\left\|Q g_{1}^{r e}(\theta) Q^{-1}\right\| \leqslant 2 \epsilon^{1-\frac{\sigma}{5}}$,

(3.17) $|F|_{r^{\prime}} \leqslant\left|Q g_{2}^{r e}(\theta) Q^{-1}\right|_{r^{\prime}} \leqslant c \epsilon^{1-\frac{11 \sigma}{5}} e^{-N^{\prime}\left(r-r^{\prime}\right)} e^{N r^{\prime}} \leqslant \epsilon_{+} \ll \epsilon^{100}$.

By (3.16) and (3.17), direct computation shows that

$$
e^{L+F(\theta)}=e^{L}+\mathcal{O}(F(\theta))=e^{L}\left(I d+e^{-L} \mathcal{O}(F(\theta))\right)=e^{L} e^{f_{+}(\theta)} .
$$

It immediately implies that

$$
\left|f_{+}(\theta)\right|_{r^{\prime}} \leqslant 2|F(\theta)|_{r^{\prime}} \leqslant 2 \epsilon_{+} \ll \epsilon^{100} .
$$

Thus we can rewrite (3.13) as

$$
B(\theta+\alpha)\left(A e^{f(\theta)}\right) B^{-1}(\theta)=A_{+} e^{f_{+}(\theta)},
$$

with

$$
A_{+}=\tilde{A}^{\prime} e^{L}=e^{A^{\prime \prime}}, \quad A^{\prime \prime} \in \operatorname{sl}(2, \mathbb{R}) .
$$


Then (3.8) and (3.16) gives

$$
\left\|A^{\prime \prime}\right\| \leqslant 2\left(\left|\rho-\frac{<n_{*}, \omega>}{2}\right|+\left\|Q g_{0}^{r e} Q^{-1}+Q g_{1}^{r e}(\theta) Q^{-1}\right\|\right) \leqslant 2 \epsilon^{\sigma} .
$$

This finishes the proof of Proposition 3.1.

3.3. Finitely differentiable case. Now we are ready to transform the estimates in the analytic case into those in the $C^{k}$ case by analytic approximation.

Let $\left(f_{j}\right)_{j \geqslant 1}, f_{j} \in C_{\frac{1}{j}}^{\omega}\left(\mathbb{T}^{d}, s l(2, \mathbb{R})\right)$ be the analytic sequence approximating $f \in C^{k}\left(\mathbb{T}^{d}, \operatorname{sl}(2, \mathbb{R})\right)$, which satisfies (2.1). Let $c, D$ be the constants defined in Proposition 3.1. Denote

$$
\epsilon_{0}^{\prime}\left(r, r^{\prime}\right)=\frac{c}{(2\|A\|)^{D}}\left(r-r^{\prime}\right)^{D \tau}
$$

Then for any $k \geqslant 5 D \tau$, one can easily compute that

$$
\frac{c}{(2\|A\|)^{D} m^{\frac{k}{4}}} \leqslant \epsilon_{0}^{\prime}\left(\frac{1}{m}, \frac{1}{m^{2}}\right)
$$

for any $m \geqslant 10, m \in \mathbb{Z}$.

We start from $m_{0}=M>\max \left\{\frac{(2\|A\|)^{D}}{c}, 10\right\}$ and denote $l_{j}=M^{2^{j-1}}, j \geqslant 1$,

$$
\epsilon_{m}=\frac{c}{(2\|A\|)^{D} m^{\frac{k}{4}}} .
$$

Proposition 3.2. Let $\alpha \in D C(\kappa, \tau), A \in S L(2, \mathbb{R}), \sigma=\frac{1}{10}, f \in C^{k}\left(\mathbb{T}^{d}\right.$, $\operatorname{sl}(2, \mathbb{R}))$ with $k \geqslant 5 D \tau$ and $f_{j}$ be as above, there exists $\epsilon_{0}=\epsilon_{0}(\kappa, \tau, d, k,\|A\|)$ such that if $\|f\|_{k} \leqslant \epsilon_{0}$, then there exist $B_{l_{j}} \in C_{\frac{1}{l_{j+1}}}^{\omega}\left(2 \mathbb{T}^{d}, S L(2, \mathbb{R})\right), A_{l_{j}} \in$ $S L(2, \mathbb{R}), f_{l_{j}}^{\prime} \in C_{\frac{1}{l_{j+1}}}^{\omega_{1}}\left(\mathbb{T}^{d}, \operatorname{sl}(2, \mathbb{R})\right)$, such that

$$
B_{l_{j}}(\theta+\alpha)\left(A e^{f_{l_{j}}(\theta)}\right) B_{l_{j}}^{-1}(\theta)=A_{l_{j}} e^{f_{l_{j}}^{\prime}(\theta)},
$$

with estimates

$$
\begin{gathered}
\left|B_{l_{j}}(\theta)\right|_{\frac{1}{l_{j+1}}} \leqslant \epsilon_{l_{j}}^{-\frac{2 \sigma}{5}}, \quad\left\|B_{l_{j}}(\theta)\right\|_{0} \leqslant \epsilon_{l_{j}}^{-\frac{\sigma}{4}}, \\
\left|f_{l_{j}}^{\prime}(\theta)\right|_{\frac{1}{l_{j+1}}} \leqslant \frac{1}{2} \epsilon_{l_{j}}^{\frac{5}{2}}, \quad\left\|A_{l_{j}}\right\| \leqslant 2\|A\| .
\end{gathered}
$$

Moreover, there exists unitary $U_{j} \in S L(2, \mathbb{C})$ such that

$$
U_{j} A_{l_{j}} U_{j}^{-1}=\left(\begin{array}{cc}
e^{\gamma_{j}} & c_{j} \\
0 & e^{-\gamma_{j}}
\end{array}\right)
$$

and

$$
\left|c_{j}\right|\left\|B_{l_{j}}(\theta)\right\|_{0}^{2} \leqslant 8\|A\|
$$


with $\gamma_{j} \in i \mathbb{R} \cup \mathbb{R}$ and $c_{j} \in \mathbb{C}$.

Proof. First step: Assume that

$$
C^{\prime}\|f(\theta)\|_{k} \leqslant \frac{c}{(2\|A\|)^{D} l_{1}^{\frac{k}{4}}}
$$

by (2.1) we have

$$
\left|f_{l_{1}}(\theta)\right|_{\frac{1}{l_{1}}} \leqslant \epsilon_{l_{1}} \leqslant \epsilon_{0}^{\prime}\left(\frac{1}{l_{1}}, \frac{1}{l_{2}}\right) .
$$

Apply Proposition 3.1, we can find $B_{l_{1}} \in C_{\frac{1}{l_{2}}}^{\omega}\left(2 \mathbb{T}^{d}, S L(2, \mathbb{R})\right), A_{l_{1}} \in S L(2, \mathbb{R})$ and $f_{l_{1}}^{\prime} \in C_{\frac{1}{l_{2}}}^{\omega}\left(\mathbb{T}^{d}, \operatorname{sl}(2, \mathbb{R})\right)$ such that

$$
B_{l_{1}}(\theta+\alpha)\left(A e^{f_{l_{1}}(\theta)}\right) B_{l_{1}}^{-1}(\theta)=A_{l_{1}} e^{f_{l_{1}}^{\prime}(\theta)},
$$

More precisely, we have two different cases:

- (Non-resonant case)

$$
\left|B_{l_{1}}\right|_{\frac{1}{l_{2}}} \leqslant 1+\epsilon_{l_{1}}^{\frac{1}{2}}, \quad\left|f_{l_{1}}^{\prime}\right|_{\frac{1}{l_{2}}} \leqslant 4 \epsilon_{l_{1}}^{3-2 \sigma} \leqslant \frac{1}{2} \epsilon_{l_{1}}^{\frac{5}{2}},
$$

and

$$
\left\|A_{l_{1}}-A\right\| \leqslant 2\|A\| \epsilon_{l_{1}}
$$

- (Resonant case)

$$
\begin{gathered}
\left|B_{l_{1}}\right|_{\frac{1}{l_{2}}} \leqslant \epsilon_{l_{1}}^{-\frac{\sigma}{10}} \times \epsilon_{l_{1}}^{\frac{-1}{l_{1}-1}} \leqslant \epsilon_{l_{1}}^{-\frac{\sigma}{5}}, \quad\left|B_{l_{1}}\right|_{0} \leqslant \epsilon_{l_{1}}^{-\frac{\sigma}{10}}, \\
\left|f_{l_{1}}^{\prime}\right|_{\frac{1}{l_{2}}} \ll \epsilon_{l_{1}}^{100} \leqslant \frac{1}{2} \epsilon_{l_{1}}^{\frac{5}{2}} .
\end{gathered}
$$

Moreover, $A_{l_{1}}=e^{A_{l_{1}}^{\prime \prime}}$ with $\left\|A_{l_{1}}^{\prime \prime}\right\| \leqslant 2 \epsilon_{l_{1}}^{\sigma}$.

Induction step: Assume that for $l_{n}, n \leqslant k$, we already have (3.22) and

$$
B_{l_{n}}(\theta+\alpha)\left(A e^{f_{l_{n}}(\theta)}\right) B_{l_{n}}^{-1}(\theta)=A_{l_{n}} e^{f_{l_{n}}^{\prime}(\theta)},
$$

with

$$
\left|B_{l_{n}}(\theta)\right|_{\frac{1}{l_{n+1}}} \leqslant \epsilon_{l_{n}}^{-\frac{2 \sigma}{5}}, \quad\left\|B_{l_{n}}(\theta)\right\|_{0} \leqslant \epsilon_{l_{n}}^{-\frac{\sigma}{4}}, \quad\left|f_{l_{n}}^{\prime}(\theta)\right|_{\frac{1}{l_{n+1}}} \leqslant \frac{1}{2} \epsilon_{l_{n}}^{\frac{5}{2}},
$$

and

$$
\left\|A_{l_{n}}\right\| \leqslant 2\|A\| \text {. }
$$

Moreover, if the $n$-th step is obtained by the resonant case, we have

$$
A_{l_{n}}=e^{A_{l_{n}}^{\prime \prime}}, \quad\left\|A_{l_{n}}^{\prime \prime}\right\|<2 \epsilon_{l_{n}}^{\sigma} .
$$

If the $n$-th step is obtained by the non-resonant case, we have

$$
\left\|A_{l_{n}}-A_{l_{n-1}}\right\| \leqslant 2\left\|A_{l_{n-1}}\right\| \epsilon_{l_{n}} .
$$

and

$$
\left\|B_{l_{n}}\right\|_{\frac{1}{l_{n+1}}} \leqslant\left(1+\epsilon_{l_{n}}^{\frac{1}{2}}\right)\left\|B_{l_{n-1}}\right\|_{\frac{1}{l_{n}}} .
$$


Now by $(3.23)$, for $l_{n+1}, n=k$, we have

$B_{l_{n}}(\theta+\alpha)\left(A e^{f_{l_{n+1}}}\right) B_{l_{n}}^{-1}(\theta)=A_{l_{n}} e^{f_{l_{n}}^{\prime}}+B_{l_{n}}(\theta+\alpha)\left(A e^{f_{l_{n+1}}}-A e^{f_{l_{n}}}\right) B_{l_{n}}^{-1}(\theta)$.

In (2.1), a simple integral implies

$$
\left|f_{l_{n+1}}(\theta)-f_{l_{n}}(\theta)\right|_{\frac{1}{l_{n+1}}} \leqslant \frac{c}{(2\|A\|)^{D} l_{n}^{k-1}}
$$

Moreover, (2.1) also gives us

$$
\left|f_{l_{n+1}}(\theta)\right|_{\frac{1}{l_{n+1}}}+\left|f_{l_{n}}(\theta)\right|_{\frac{1}{l_{n+1}}} \leqslant \frac{2 c}{(2\|A\|)^{D} M^{\frac{k}{4}}} .
$$

Thus if we rewrite that

$$
A_{l_{n}} e^{f_{l_{n}}^{\prime}(\theta)}+B_{l_{n}}(\theta+\alpha)\left(A e^{f_{l_{n+1}}(\theta)}-A e^{f_{l_{n}}(\theta)}\right) B_{l_{n}}^{-1}(\theta)=A_{l_{n}} e^{\widetilde{f_{l_{n}}}(\theta)},
$$

by $(3.24),(3.25),(3.29)$ and (3.30) we obtain

$$
\begin{aligned}
\left|\widetilde{f_{l_{n}}}(\theta)\right|_{\frac{1}{l_{n+1}}} & \leqslant\left|f_{l_{n}}^{\prime}(\theta)\right|_{\frac{1}{l_{n+1}}}+\left\|A_{l_{n}}^{-1}\right\|\left|B_{l_{n}}(\theta+\alpha)\left(A e^{f_{l_{n+1}}(\theta)}-A e^{f_{l_{n}}(\theta)}\right) B_{l_{n}}^{-1}(\theta)\right|_{\frac{1}{l_{n+1}}} \\
& \leqslant \frac{1}{2} \epsilon_{l_{n}}^{\frac{5}{2}}+2\|A\|^{2} \times \epsilon_{l_{n}}^{-\frac{4 \sigma}{5}} \times \frac{c}{(2\|A\|)^{D} l_{n}^{k-1}} \\
& \leqslant \frac{1}{2} \epsilon_{l_{n+1}}+\frac{1}{2} \times \frac{c}{(2\|A\|)^{D} l_{n}^{\frac{k}{2}}} \\
& \leqslant \epsilon_{l_{n+1}} \\
& \leqslant \epsilon_{0}^{\prime}\left(\frac{1}{l_{n+1}}, \frac{1}{l_{n+2}}\right) .
\end{aligned}
$$

Now for $\left(\alpha, A_{l_{n}} e^{\widetilde{f_{l_{n}}}(\theta)}\right)$, we can apply Proposition 3.1 again to get $\tilde{B}_{l_{n}} \in$ $C_{\frac{1}{l_{n+2}}}^{\omega}\left(2 \mathbb{T}^{d}, S L(2, \mathbb{R})\right), A_{l_{n+1}} \in S L(2, \mathbb{R})$ and $f_{l_{n+1}}^{\prime} \in C_{\frac{1}{l_{n+2}}}^{\omega}\left(\mathbb{T}^{d}, \operatorname{sl}(2, \mathbb{R})\right)$ such that

$$
\tilde{B}_{l_{n}}(\theta+\alpha)\left(A_{l_{n}} e^{\widetilde{f_{l_{n}}}(\theta)}\right) \tilde{B}_{l_{n}}(\theta)=A_{l_{n+1}} e^{f_{l_{n+1}}^{\prime}(\theta)}
$$

with

$$
\left|\tilde{B}_{l_{n}}(\theta)\right|_{\frac{1}{l_{n+2}}} \leqslant \epsilon_{l_{n+1}}^{-\frac{\sigma}{10}} \times \epsilon_{l_{n+1}}^{\frac{-1}{l_{n+1}-1}}, \quad\left\|\tilde{B}_{l_{n}}(\theta)\right\|_{0} \leqslant \epsilon_{l_{n+1}}^{-\frac{\sigma}{10}}, \quad\left|f_{l_{n+1}}^{\prime}(\theta)\right|_{\frac{1}{l_{n+2}}} \leqslant \frac{1}{2} \epsilon_{l_{n+1}}^{\frac{5}{2}} .
$$

Denote $B_{l_{n+1}}:=\tilde{B}_{l_{n}} B_{l_{n}} \in C_{\frac{1}{l_{n+2}}}^{\omega}\left(2 \mathbb{T}^{d}, S L(2, \mathbb{R})\right)$, then

$$
\left|B_{l_{n+1}}(\theta)\right|_{\frac{1}{l_{n+2}}} \leqslant \epsilon_{l_{n}}^{-\frac{2 \sigma}{5}} \times \epsilon_{l_{n+1}}^{-\frac{\sigma}{10}} \times \epsilon_{l_{n+1}}^{\frac{-1}{l_{n+1}-1}} \leqslant \epsilon_{l_{n+1}}^{-\frac{2 \sigma}{5}},
$$

and

$$
\left\|B_{l_{n+1}}(\theta)\right\|_{0} \leqslant \epsilon_{l_{n}}^{-\frac{\sigma}{4}} \times \epsilon_{l_{n+1}}^{-\frac{\sigma}{10}} \leqslant \epsilon_{l_{n+1}}^{-\frac{\sigma}{4}} .
$$

For the remaining estimates, we distinguish two cases.

- If the $(n+1)$-th step is in the resonant case, we have

$$
A_{l_{n+1}}=e^{A_{l_{n+1}}^{\prime \prime}}, \quad\left\|A_{l_{n+1}}^{\prime \prime}\right\|<2 \epsilon_{l_{n+1}}^{\sigma}, \quad\left\|A_{l_{n+1}}\right\| \leqslant 1+2 \epsilon_{l_{n+1}}^{\sigma} \leqslant 2\|A\| .
$$


Then there exists unitary $U \in S L(2, \mathbb{C})$ such that

$$
U A_{l_{n+1}} U^{-1}=\left(\begin{array}{cc}
e^{\gamma_{n+1}} & c_{n+1} \\
0 & e^{-\gamma_{n+1}}
\end{array}\right)
$$

with $\left|c_{n+1}\right| \leqslant 2\left\|A_{l_{n+1}}^{\prime \prime}\right\| \leqslant 4 \epsilon_{l_{n+1}}^{\sigma}$. Thus (3.22) is fulfilled.

- If the $(n+1)$-th step is in the non-resonant case, one traces back to the resonant step $j$ which is closest to $n+1$.

If $j$ exists, by (3.24) and (3.26) we have

$$
\begin{gathered}
\left|B_{l_{j}}(\theta)\right|_{\frac{1}{l_{j+1}}} \leqslant \epsilon_{l_{j}}^{-\frac{2 \sigma}{5}}, \quad\left\|B_{l_{j}}(\theta)\right\|_{0} \leqslant \epsilon_{l_{j}}^{-\frac{\sigma}{4}}, \\
A_{l_{j}}=e^{A_{l_{j}}^{\prime \prime}}, \quad\left\|A_{l_{j}}^{\prime \prime}\right\|<2 \epsilon_{l_{j}}^{\sigma}, \quad\left\|A_{l_{j}}\right\| \leqslant 1+2 \epsilon_{l_{j}}^{\sigma} .
\end{gathered}
$$

By our choice of $j$, from $j$ to $n+1$, every step is non-resonant. Thus by (3.27) we obtain

$$
\left\|A_{l_{n+1}}-A_{l_{j}}\right\| \leqslant 2 \epsilon_{l_{j}}^{\frac{1}{2}}
$$

SO

$$
\left\|A_{l_{n+1}}\right\| \leqslant 1+2 \epsilon_{l_{j}}^{\sigma}+2 \epsilon_{l_{j}}^{\frac{1}{2}} \leqslant 2\|A\| .
$$

Estimate (3.32) implies that if we rewrite $A_{l_{n+1}}=e^{A_{l_{n+1}}^{\prime \prime}}$, then

$$
\left\|A_{l_{n+1}}^{\prime \prime}\right\| \leqslant 4 \epsilon_{l_{j}}^{\sigma} \text {. }
$$

Moreover, by (3.28), we have

$$
\left\|B_{l_{n+1}}(\theta)\right\|_{0} \leqslant\left\|B_{l_{n+1}}(\theta)\right\|_{\frac{1}{l_{n+1}}} \leqslant 2\left|B_{l_{j}}(\theta)\right|_{\frac{1}{l_{j+1}}} \leqslant 2 \epsilon_{l_{j}}^{-\frac{2 \sigma}{5}} .
$$

Similarly to the process of (3.31), (3.22) is fulfilled.

If $j$ vanishes, it immediately implies that from 1 to $n+1$, each step is nonresonant. In this case, $\left\|A_{l_{n+1}}\right\| \leqslant 2\|A\|$ and the estimate (3.22) is naturally satisfied as

$$
\left\|B_{l_{n+1}}(\theta)\right\|_{\frac{1}{l_{n+1}}} \leqslant 2
$$

3.4. Differentiable almost reducibility. Now, we are ready to show the quantitative almost reducibility for $C^{k}$ quasi-periodic $S L(2, \mathbb{R})$ cocycles.

Theorem 3.1. Let $\alpha \in D C(\kappa, \tau), A \in S L(2, \mathbb{R}), \sigma=\frac{1}{10}, f \in C^{k}\left(\mathbb{T}^{d}, \operatorname{sl}(2, \mathbb{R})\right)$ with $k \geqslant 5 D \tau$, there exists $\epsilon_{1}=\epsilon_{1}(\kappa, \tau, d, k,\|A\|)$ such that if $\|f\|_{k} \leqslant \epsilon_{1}$ then $\left(\alpha, A e^{f(\theta)}\right)$ is $C^{k, k_{0}}$ almost reducible with $k_{0} \in \mathbb{N}, k_{0} \leqslant \frac{k}{6}$. Moreover, if we further assume $\left(\alpha, A e^{f(\theta)}\right)$ is not uniformly hyperbolic, then there exists $B_{l_{j}} \in C_{\frac{1}{l_{j+1}}}^{\omega}\left(2 \mathbb{T}^{d}, S L(2, \mathbb{C})\right), A_{l_{j}} \in S L(2, \mathbb{C}), \tilde{F}_{l_{j}} \in C^{k}\left(\mathbb{T}^{d}, S L(2, \mathbb{C})\right)$, such that

$$
B_{l_{j}}(\theta+\alpha)\left(A e^{f(\theta)}\right) B_{l_{j}}^{-1}(\theta)=A_{l_{j}}+\tilde{F}_{l_{j}}(\theta)
$$


with

$$
\left\|B_{l_{j}}\right\|_{0} \leqslant \epsilon_{l_{j}}^{-\frac{\sigma}{4}}, \quad\left\|\tilde{F}_{l_{j}}\right\|_{0} \leqslant \epsilon_{l_{j}}^{\frac{1}{4}}
$$

and $A_{l_{j}}=\left(\begin{array}{cc}e^{i \gamma_{j}} & c_{j} \\ 0 & e^{-i \gamma_{j}}\end{array}\right)$ with estimate

$$
\left\|B_{l_{j}}\right\|_{0}^{2}\left|c_{j}\right| \leqslant 8\|A\|
$$

where $\gamma_{j} \in \mathbb{R}$ and $c_{j} \in \mathbb{C}$.

Remark 3.2. Estimate (3.33) is essential for us to obtain 1/2-Hölder continuity.

Proof. We first deal with the $C^{0}$ estimate as it is much more significant for the proof of our main Theorem. By Proposition 3.2, we have for any $l_{j}$, $j \in \mathbb{N}^{+}$:

$$
B_{l_{j}}(\theta+\alpha)\left(A e^{f_{l_{j}}(\theta)}\right) B_{l_{j}}^{-1}(\theta)=A_{l_{j}} e^{f_{l_{j}}^{\prime}(\theta)}
$$

thus

$B_{l_{j}}(\theta+\alpha)\left(A e^{f(\theta)}\right) B_{l_{j}}^{-1}(\theta)=A_{l_{j}} e^{f_{l_{j}}^{\prime}(\theta)}+B_{l_{j}}(\theta+\alpha)\left(A e^{f(\theta)}-A e^{f_{l_{j}}(\theta)}\right) B_{l_{j}}^{-1}(\theta)$.

Denote

$$
A_{l_{j}}+\tilde{F}_{l_{j}}(\theta)=A_{l_{j}} e^{f_{l_{j}}^{\prime}(\theta)}+B_{l_{j}}(\theta+\alpha)\left(A e^{f(\theta)}-A e^{f_{l_{j}}(\theta)}\right) B_{l_{j}}^{-1}(\theta) .
$$

In (2.1), by a simple integration we get

$$
\left\|f(\theta)-f_{l_{j}}(\theta)\right\|_{0} \leqslant \frac{c}{(2\|A\|)^{D} l_{j}^{k-1}},
$$

and

$$
\|f(\theta)\|_{0}+\left\|f_{l_{j}}(\theta)\right\|_{0} \leqslant \frac{c}{(2\|A\|)^{D} M^{\frac{k}{4}}}+\frac{c}{(2\|A\|)^{D} C^{\prime} M^{\frac{k}{4}}} .
$$

Proposition 3.2 also gives the estimates

$$
\left\|B_{l_{j}}(\theta)\right\|_{0} \leqslant \epsilon_{l_{j}}^{-\frac{\sigma}{4}}, \quad\left|f_{l_{j}}^{\prime}(\theta)\right|_{\frac{1}{l_{j+1}}} \leqslant \frac{1}{2} \epsilon_{l_{j}}^{\frac{5}{2}},
$$

and

$$
\left\|A_{l_{j}}\right\| \leqslant 2\|A\|
$$

Thus by $(3.34)-(3.38)$, we have

$$
\begin{aligned}
\left\|\tilde{F}_{l_{j}}(\theta)\right\|_{0} & \leqslant\left\|A_{l_{j}} f_{l_{j}}^{\prime}(\theta)\right\|_{0}+\left\|B_{l_{j}}(\theta+\alpha)\left(A e^{f(\theta)}-A e^{f_{l_{j}}(\theta)}\right) B_{l_{j}}^{-1}(\theta)\right\|_{0} \\
& \leqslant\|A\| \epsilon_{l_{j}}^{\frac{5}{2}}+\|A\| \times \epsilon_{l_{j}}^{-\frac{\sigma}{2}} \times \frac{c}{(2\|A\|)^{D} l_{j}^{k-1}} \leqslant \frac{\epsilon_{l_{j}}^{2}}{2\|A\|} .
\end{aligned}
$$

To prove (3.33), Proposition 3.2 implies that we only need to exclude the case when $\gamma_{j} \in i \mathbb{R} \backslash\{0\}$. 
We assume that $\operatorname{spec}\left(A_{l_{j}}\right)=\left\{e^{\lambda_{j}}, e^{-\lambda_{j}}\right\}, \lambda_{j} \in \mathbb{R} \backslash\{0\}$, thus we can always find $P \in S O(2, \mathbb{R})$ such that

$$
P A_{l_{j}} P^{-1}=\left(\begin{array}{cc}
e^{\lambda_{j}} & c_{j} \\
0 & e^{-\lambda_{j}}
\end{array}\right)
$$

with $\left|c_{j}\right| \leqslant\left\|A_{l_{j}}\right\| \leqslant 2\|A\|$.

If $\left|\lambda_{j}\right|>\epsilon_{l_{j}}^{\frac{1}{4}}$, set $B=\operatorname{diag}\left\{\|2 A\|^{-\frac{1}{2}} \epsilon_{l_{j}}^{\frac{1}{2}},\|2 A\|^{\frac{1}{2}} \epsilon_{l_{j}}^{-\frac{1}{2}}\right\}$, then

$$
B P\left(A_{l_{j}}+\tilde{F}_{l_{j}}(\theta)\right) P^{-1} B^{-1}=\left(\begin{array}{cc}
e^{\lambda_{j}} & 0 \\
0 & e^{-\lambda_{j}}
\end{array}\right)+F(\theta),
$$

where $\|F(\theta)\|_{0} \leqslant 2 \epsilon_{l_{j}}$. We rewrite

$$
\left(\begin{array}{cc}
e^{\lambda_{j}} & 0 \\
0 & e^{-\lambda_{j}}
\end{array}\right)+F(\theta)=\left(\begin{array}{cc}
e^{\lambda_{j}} & 0 \\
0 & e^{-\lambda_{j}}
\end{array}\right) e^{\tilde{f}(\theta)}
$$

with $\|\tilde{f}(\theta)\|_{0} \leqslant 4 \epsilon_{l_{j}}$. Then by Remark 3.1 and Corollary 3.1 of [24], one can conjugate (3.39) to

$$
\left(\begin{array}{cc}
e^{\lambda_{j}} & 0 \\
0 & e^{-\lambda_{j}}
\end{array}\right)\left(\begin{array}{cc}
e^{\tilde{f}^{r e}(\theta)} & 0 \\
0 & e^{-\tilde{f}^{r e}(\theta)}
\end{array}\right)=\left(\begin{array}{cc}
e^{\lambda_{j}} e^{\tilde{f}^{r e}(\theta)} & 0 \\
0 & e^{-\lambda_{j}} e^{-\tilde{f}^{r e}(\theta)}
\end{array}\right)
$$

with $\left\|\tilde{f}^{r e}(\theta)\right\|_{0} \leqslant 8 \epsilon_{l_{j}}$, thus $\left(\alpha, A e^{f(\theta)}\right)$ is uniformly hyperbolic, which contradicts our assumption. Thus we only need to consider $\left|\lambda_{j}\right| \leqslant \epsilon_{l_{j}}^{\frac{1}{4}}$; in this case, we put $\lambda_{j}$ into the perturbation so that the new perturbation satisfies $\left\|\tilde{F}_{l_{j}}^{\prime}\right\|_{0} \leqslant \epsilon_{l_{j}}^{\frac{1}{4}}$ and

$$
A_{l_{j}}=\left(\begin{array}{cc}
1 & c_{j} \\
0 & 1
\end{array}\right)
$$

Although $C^{0}$ norm is sufficient to prove our main Theorem, we want to show the actually differentiable almost reducibility instead of $C^{0}$ almost reducibility. By Cauchy estimates, for $k_{0} \in \mathbb{N}$ and $k_{0} \leqslant k$, we have

$$
\begin{aligned}
\left\|f_{j}-f_{j+1}\right\|_{k_{0}} & \leqslant \sup _{l \leqslant k_{0}, \theta \in \mathbb{T}^{d}}\left\|\left(\partial_{\theta_{1}}^{l_{1}}+\cdots+\partial_{\theta_{d}}^{l_{d}}\right)\left(f_{j}(\theta)-f_{j+1}(\theta)\right)\right\| \\
& \leqslant\left(k_{0}\right) !(j+1)^{k_{0}}\left|f_{j}-f_{j+1}\right|_{\frac{1}{j+1}} \\
& \leqslant\left(k_{0}\right) !(j+1)^{k_{0}} \times \frac{c}{(2\|A\|)^{D} j^{k}} \\
& \leqslant \frac{C_{1}}{j^{k-k_{0}}}
\end{aligned}
$$

where $C_{1}$ is independent of $j$.

By a simple integration we get

$$
\left\|f(\theta)-f_{l_{j}}(\theta)\right\|_{k_{0}} \leqslant \frac{C_{1}}{l_{j}^{k-k_{0}-1}} .
$$


Similarly by Cauchy estimates, we have

$$
\begin{aligned}
\left\|f_{l_{j}}^{\prime}(\theta)\right\|_{k_{0}} & \leqslant\left(k_{0}\right) !\left(l_{j+1}\right)^{k_{0}}\left|f_{l_{j}}^{\prime}(\theta)\right| \frac{1}{l_{j+1}} \\
& \leqslant\left(k_{0}\right) !\left(l_{j}\right)^{2 k_{0}} \times \frac{1}{2} \epsilon_{l_{j}}^{\frac{5}{2}} \\
& \leqslant\left(k_{0}\right) !\left(l_{j}\right)^{2 k_{0}} \times \frac{1}{2} \times\left(\frac{c}{(2\|A\|)^{D} l_{j}^{\frac{k}{4}}}\right)^{\frac{5}{2}} \\
& \leqslant \frac{C_{2}}{l_{j}^{\frac{5 k}{8}-2 k_{0}}}
\end{aligned}
$$

where $C_{2}$ is independent of $j$.

$$
\begin{aligned}
\left\|B_{l_{j}}(\theta)\right\|_{k_{0}} & \leqslant\left(k_{0}\right) !\left(l_{j+1}\right)^{k_{0}}\left|B_{l_{j}}(\theta)\right|_{\frac{1}{l_{j+1}}} \\
& \leqslant\left(k_{0}\right) !\left(l_{j}\right)^{2 k_{0}} \times \epsilon_{l_{j}}^{-\frac{2 \sigma}{5}} \\
& \leqslant\left(k_{0}\right) !\left(l_{j}\right)^{2 k_{0}} \times\left(\frac{c}{(2\|A\|)^{D} l_{j}^{\frac{k}{4}}}\right)^{-\frac{2 \sigma}{5}} \\
& \leqslant C_{3} l_{j}^{\frac{k}{100}+2 k_{0}}
\end{aligned}
$$

where $C_{3}$ is independent of $j$. Thus we have

$$
\begin{aligned}
\left\|\tilde{F}_{l_{j}}(\theta)\right\|_{k_{0}} & \leqslant\left\|A_{l_{j}} f_{l_{j}}^{\prime}(\theta)\right\|_{k_{0}}+\left\|B_{l_{j}}(\theta+\alpha)\left(A e^{f(\theta)}-A e^{f_{l_{j}}(\theta)}\right) B_{l_{j}}^{-1}(\theta)\right\|_{k_{0}} \\
& \leqslant \frac{C_{4}}{l_{j}^{\frac{5 k}{8}-2 k_{0}}}+C_{5} l_{j}^{\frac{k}{50}+4 k_{0}} \times l_{j}^{-k+k_{0}+1} \\
& \leqslant \frac{C_{4}}{l_{j}^{\frac{5 k}{8}-2 k_{0}}}+\frac{C_{5}}{l_{j}^{\frac{49 k}{50}-5 k_{0}-1}} .
\end{aligned}
$$

If $k_{0} \leqslant \frac{k}{6}$, then

$$
\left\|\tilde{F}_{l_{j}}(\theta)\right\|_{k_{0}} \leqslant \frac{C_{6}}{l_{j}^{2}},
$$

which immediately shows that $\left\{\tilde{F}_{l_{j}}\right\}_{j \geqslant 1}$ is a Cauchy sequence in $C^{k_{0}}$ topology with

$$
\lim _{j \rightarrow+\infty}\left\|\tilde{F}_{l_{j}}(\theta)\right\|_{k_{0}}=0
$$

which means precisely that $\left(\alpha, A e^{f(\theta)}\right)$ is $C^{k, k_{0}}$ almost reducible.

This finishes the proof of Theorem 3.1.

3.5. Remarks on differentiable almost reducibility. The statement on differentiable almost reducibility was given in [18], based on the almost reducibility result in [17]. However, the proof in [18] is not precise since it was based on estimates on $B_{j}$ given in [17] which are not good enough. In fact, from the estimates in [17], one gets the following estimates for the change of variables $B_{j}$ (as is usual in the analytic case): 


$$
\left|B_{j}\right|_{r_{j}} \leq\left(\epsilon_{0}^{-2^{j}}\right)^{\frac{1}{4}\left(r_{j-1}-r_{j}\right)}
$$

where $r_{j}$ are the successive radii of analyticity. However, since the perturbation comes from the analytic approximation (2.1), which is of size $\frac{1}{r_{j-1}^{k}}$, when $r_{j}$ decreases as $\frac{1}{j}$ as in [17]. Thus the bounds for the new perturbation is

$$
\left(\epsilon_{0}^{-2^{j}}\right)^{\frac{1}{4}\left(r_{j-1}-r_{j}\right)} \frac{1}{r_{j-1}^{k}}
$$

which is divergent, and thus the iteration can not go on. For this reason, in this paper, we have to work out for $B_{j}$ a better estimates

$$
\left(\epsilon_{j-1}\right)^{\frac{-r_{j}}{r_{j-1}-r_{j}}}
$$

which is enough for our purposes.

Another improvement is the lower bound on the degree of differentiability $k$ (we obtain an integer $k$ which only depends on $\tau$, which fits the intuition from classical KAM theory), motivated by the estimate (3.19) above, which is more explicit than the one in [18].

However, we do not claim optimality of the condition we impose on $k$ and $k_{0}$. In fact, our KAM scheme still has quite a lot of flexibility. As we pointed out, the new perturbation is of size $\frac{1}{r_{j-1}^{k}}$, where we have set the sequence $r_{j}=r_{j-1}^{2}$, which is more suitable for the resonant case. This has the advantage of providing a unified argument, but is clearly not optimal in the non-resonant case, since in this case, the conjugacy map is close to identity, which allows slow shrinking of the analytic radius (for example, from $1 / j$ to $1 /(j+1)$. More precise estimates should be obtainable by interpolating these two different arguments. These further modifications will have two advantages: the first is that one can obtain $C^{k, k-D}$ almost reducibility results with $D$ independent of $k$; the second advantage is that one can obtain quite better estimates on the lower bound on the degree of differentiability $k$. However, since the main point of this paper is to obtain the Hölder continuity of LE, this rough estimate is already enough for our purposes.

\section{Proof of the main Results}

As we mentioned in the introduction, nice quantitative almost reducibility of $(\alpha, A)$ easily implies Hölder continuity of the Lyapunov exponent, where "nice" means that the norm of the conjugation map is well controlled compared with the upper triangular element of the constant matrix and the norm of the perturbation. In fact, for $B$ close to $A$, we can write that $(\alpha, B)=(\alpha, A+(B-A))$, so nice quantitative almost reducibility of $(\alpha, A)$ implies nice quantitative almost reducibility of $(\alpha, B)$, which indicates the closeness between $L(\alpha, B)$ and $L(\alpha, A)$, as we shall see below. 


\subsection{Proof of Theorem 1.1.}

Proof. If the cocycle is uniformly hyperbolic, then the Lyapunov exponent is Lipschitz, which follows from the cone-field criterion. Therefore, we only need to consider the case $(\alpha, \mathcal{A})$ is almost reducible, but not uniformly hyperbolic.

By the definition of $C^{k^{\prime}, k}$ almost reducibility, there exist $B_{j} \in C^{k}\left(\mathbb{T}^{d}\right.$, $S L(2, \mathbb{R})), A \in S L(2, \mathbb{R})$ and $F_{j} \in C^{k}\left(\mathbb{T}^{d}, \operatorname{sl}(2, \mathbb{R})\right)$ such that

$$
B_{j}(\theta+\alpha) \mathcal{A}(\theta) B_{j}(\theta)^{-1}=A e^{F_{j}(\theta)}
$$

with $\left\|F_{j}\right\|_{k} \leqslant \epsilon_{j} \rightarrow 0$. Furthermore, without loss of generality, we may assume $\|A\| \leqslant 1$.

Now we have $\|A\| \leqslant 1$ and $k \geqslant D_{0} \tau=5 D \tau$. In the following, $C_{0}$ is a large constant depending on $d, \kappa, \tau$ and $c$ is a small constant depending on $d, \kappa, \tau$. Notice that $\left(\alpha, A e^{F_{j}(\theta)}\right)$ is not uniformly hyperbolic, so for $j$ large enough, it will finally fall into our local regime in Theorem 3.1. By Theorem 3.1, we rewrite

$$
A_{l_{j}}+\tilde{F}_{l_{j}}(\theta)=\left(\begin{array}{cc}
e^{i \gamma_{j}} & 0 \\
0 & e^{-i \gamma_{j}}
\end{array}\right)+\left(\begin{array}{cc}
q_{1}(\theta) & q_{2}(\theta) \\
q_{3}(\theta) & q_{4}(\theta)
\end{array}\right)
$$

where

$$
\begin{gathered}
\left\|q_{2}(\theta)\right\|_{0} \leqslant\left|c_{j}\right|+\left\|\tilde{F}_{l_{j}}\right\|_{0} \leqslant\left|c_{j}\right|+\epsilon_{l_{j}}^{\frac{1}{4}}, \\
\left\|q_{1}(\theta)\right\|_{0},\left\|q_{3}(\theta)\right\|_{0},\left\|q_{4}(\theta)\right\|_{0} \leqslant 2 \epsilon_{l_{j}}^{\frac{1}{4}} \leqslant \frac{C_{0}}{l_{j}^{\frac{k}{16}}} .
\end{gathered}
$$

Let

$$
D=\left(\begin{array}{cc}
d & 0 \\
0 & d^{-1}
\end{array}\right)
$$

where $d=\left\|B_{l_{j}}\right\|_{0} \epsilon^{\frac{1}{4}}$. Let $W(\theta)=D B_{l_{j}}(\theta)$. If $d \leqslant 1$, i.e.

$$
\epsilon \leqslant\left(\epsilon_{l_{j}}^{-\frac{\sigma}{4}}\right)^{-4}
$$

thus we pick

$$
\epsilon \leqslant c l_{j}^{-\frac{k}{40}}
$$

then we have

$$
\|W\|_{0} \leqslant C_{0} \epsilon^{-\frac{1}{4}}
$$

Since

$$
D\left(\begin{array}{ll}
x_{1} & x_{2} \\
x_{3} & x_{4}
\end{array}\right) D^{-1}=\left(\begin{array}{cc}
x_{1} & d^{2} x_{2} \\
d^{-2} x_{3} & x_{4}
\end{array}\right)
$$

we have

$$
Z(\theta)=W(\theta+\alpha)\left(A e^{f}\right) W(\theta)^{-1}=\left(\begin{array}{cc}
e^{i \gamma_{j}} & 0 \\
0 & e^{-i \gamma_{j}}
\end{array}\right)+\left(\begin{array}{cc}
q_{1}(\theta) & d^{2} q_{2}(\theta) \\
d^{-2} q_{3}(\theta) & q_{4}(\theta)
\end{array}\right)
$$

with

$$
\left\|q_{1}\right\|_{0},\left\|q_{4}\right\|_{0},\left\|d^{-2} q_{3}\right\|_{0} \leqslant C_{0} \epsilon^{-\frac{1}{2}} l_{j}^{-\frac{k}{16}}
$$


and by (3.33) we have

$$
\left\|d^{2} q_{2}\right\|_{0} \leqslant\left\|B_{l_{j}}\right\|_{0}^{2} \epsilon^{\frac{1}{2}} \times\left(\left|c_{j}\right|+\epsilon_{l_{j}}^{\frac{1}{4}}\right) \leqslant C_{0} \epsilon^{\frac{1}{2}} .
$$

Therefore, if we pick

$$
\epsilon \geqslant C_{0} l_{j}^{-\frac{k}{16}}
$$

then

$$
\|Z\|_{0} \leqslant 1+C_{0} \epsilon^{\frac{1}{2}}
$$

Now we can prove our main theorem. Notice that it is enough to consider the case when $\epsilon=\|\mathcal{B}-\mathcal{A}\|_{0}$ is sufficiently small. Then $\epsilon$ satisfies

$$
I_{j}: C_{0} l_{j}^{-\frac{k}{16}} \leqslant \epsilon \leqslant c l_{j}^{-\frac{k}{40}}
$$

for some choice of $j$ and the following inequality is fulfilled:

$$
C_{0} l_{j}^{-\frac{k}{16}} \leqslant c l_{j}^{-\frac{k}{20}}=c l_{j+1}^{-\frac{k}{40}} .
$$

This is essential as we are able to cover all the small $\epsilon$ tending to zero by the interval $I_{j}$ above.

Let

$$
\tilde{\mathcal{B}}(\theta)=W(\theta+\alpha) \mathcal{B}(\theta) W(\theta)^{-1}
$$

then

$$
\|\tilde{\mathcal{B}}\|_{0} \leqslant\|Z\|_{0}+\|W\|_{0}^{2}\|\mathcal{B}-\mathcal{A}\|_{0} \leqslant 1+C_{0} \epsilon^{\frac{1}{2}}
$$

Thus

$$
L(\alpha, \mathcal{B})=L(\alpha, \tilde{\mathcal{B}}) \leqslant \ln \|\tilde{\mathcal{B}}\|_{0} \leqslant C_{0} \epsilon^{\frac{1}{2}}
$$

Respectively,

$$
L(\alpha, \mathcal{A})=L(\alpha, Z) \leqslant \ln \|Z\|_{0} \leqslant C_{0} \epsilon^{\frac{1}{2}}
$$

It implies that

$$
|L(\alpha, \mathcal{A})-L(\alpha, \mathcal{B})| \leqslant C_{0}\|\mathcal{B}-\mathcal{A}\|_{0}^{\frac{1}{2}}
$$

where $C_{0}$ depends on $d, \kappa, \tau$. This finishes the proof of Theorem 1.1.

\subsection{Proof of Theorem 1.2.}

Proof. We rewrite the Schrödinger cocycle as

$$
S_{E}^{\lambda V}=A+F(\theta)
$$

where

$$
A=\left(\begin{array}{cc}
-E & -1 \\
1 & 0
\end{array}\right), \quad F(\theta)=\left(\begin{array}{cc}
\lambda V(\theta) & 0 \\
0 & 0
\end{array}\right) .
$$

Notice that we only need to consider

$$
E \in \Sigma_{\alpha, \lambda V} \subset[-2+\inf \lambda V, 2+\sup \lambda V],
$$

otherwise $S_{E}^{\lambda V}$ is uniformly hyperbolic and $L(E)$ (thus $N_{\lambda V, \alpha}(E)$ ) is automatically $\frac{1}{2}$-Hölder continuous. So by the assumption on $\lambda$, we have $\|A\| \leqslant 3$ 
and so does $A^{-1}$. Clearly, the smallness condition on $\lambda$ is namely the smallness condition on $F$. Thus if we write that

$$
A+F(\theta)=A e^{f(\theta)}
$$

the assumptions of Theorem 3.1 are naturally fulfilled as

$$
\|f(\theta)\|_{k} \leqslant\left\|A^{-1}\right\|\|F(\theta)\|_{k} .
$$

Therefore $C^{k, k_{0}}$ almost reducibility of $\left(\alpha, S_{E}^{\lambda V}\right)$ is a straight result of Theorem 3.1. As a corollary of Theorem 1.1, we obtain that

$$
L(E+i \epsilon)-L(E) \leqslant C_{0} \epsilon^{\frac{1}{2}}, \quad E \in \Sigma,
$$

where $C_{0}$ does not depend on $E$.

By the Thouless formula

$$
L(E)=\int \ln \left|E-E^{\prime}\right| d N_{\lambda V, \alpha}\left(E^{\prime}\right)
$$

we have

$$
\begin{aligned}
L(E+i \epsilon)-L(E) & =\frac{1}{2} \int \ln \left(1+\frac{\epsilon^{2}}{\left(E-E^{\prime}\right)^{2}}\right) d N_{\lambda V, \alpha}\left(E^{\prime}\right) \\
& \geqslant c^{\prime}\left(N_{\lambda V, \alpha}(E+\epsilon)-N_{\lambda V, \alpha}(E-\epsilon)\right)
\end{aligned}
$$

for every $\epsilon>0$ and $c^{\prime}$ is a numerical constant. Thus

$$
N_{\lambda V, \alpha}(E+\epsilon)-N_{\lambda V, \alpha}(E-\epsilon) \leqslant \frac{C_{0}}{c^{\prime}} \epsilon^{\frac{1}{2}}
$$

for every $\epsilon>0, E \in \Sigma$. Notice that $N_{\lambda V, \alpha}$ is locally constant in the gaps, this means precisely that $N_{\lambda V, \alpha}$ is $\frac{1}{2}$-Hölder continuous. This finishes the proof of Theorem 1.2.

\section{Appendix: Proof of Lemma 3.1}

In this appendix, we prove Lemma 3.1 by the following quantitative Implicit Function Theorem:

Theorem 5.1. [10, 19] Let $X, Y, Z$ be Banach spaces, $U \subset X$ and $V \subset Y$ neighborhoods of $x_{0}$ and $y_{0}$ respectively. Fix $s, \delta>0$ and define $B_{s}\left(x_{0}\right)=$ $\left\{x \in X \mid\left\|x-x_{0}\right\| \leqslant s\right\}, B_{\delta}\left(y_{0}\right)=\left\{y \in Y \mid\left\|y-y_{0}\right\| \leqslant \delta\right\}$. Let $\Psi \in$ $C^{1}(U \times V, Z)$ and $B_{s}\left(x_{0}\right) \times B_{\delta}\left(y_{0}\right) \subset U \times V$. Suppose also that $\Psi\left(x_{0}, y_{0}\right)=0$, and that $D_{y} \Psi\left(x_{0}, y_{0}\right) \in \mathcal{L}(Y, Z)$ is invertible. If

$$
\begin{gathered}
\frac{\sup }{B_{s}\left(x_{0}\right)}\left\|\Psi\left(x, y_{0}\right)\right\|_{Z} \leqslant \frac{\delta}{2\left\|\left(D_{y} \Psi\left(x_{0}, y_{0}\right)^{-1}\right)\right\|}, \\
\sup _{\overline{B_{s}\left(x_{0}\right)} \times \overline{B_{\delta}\left(y_{0}\right)}}\left\|I d_{Y}-\left(D_{y} \Psi\left(x_{0}, y_{0}\right)\right)^{-1} D_{y} \Psi(x, y)\right\|_{\mathcal{L}(Y, Y)} \leqslant \frac{1}{2},
\end{gathered}
$$

then there exists $y \in C^{1}\left(B_{s}\left(x_{0}\right), \overline{B_{\delta}\left(y_{0}\right)}\right)$ such that $\Psi(x, y(x))=0$. 
With Theorem 5.1 in hand, now we can prove Lemma 3.1 easily. We construct the nonlinear functional

$$
\Psi: \mathcal{B}_{r}^{n r e}(\eta) \times C_{r}^{\omega}\left(\mathbb{T}^{d}, s u(1,1)\right) \rightarrow \mathcal{B}_{r}^{\text {nre }}(\eta)
$$

by

$$
\Psi(Y, g)=\mathbb{P}_{n r e} \ln \left(e^{A^{-1} Y(\theta+\alpha) A} e^{g(\theta)} e^{-Y(\theta)}\right)
$$

An immediate check reveals that

$$
\Psi(0,0)=0, \quad\|\Psi(0, g)\| \leqslant|g|_{r}
$$

and

$$
\begin{aligned}
& \Psi\left(Y+Y^{\prime}, g\right)-\Psi(Y, g) \\
= & \mathbb{P}_{n r e} \ln \left(e^{A^{-1}\left(Y(\theta+\alpha)+Y^{\prime}(\theta+\alpha)\right) A} e^{g(\theta)} e^{-\left(Y(\theta)+Y^{\prime}(\theta)\right)}\right) \\
& -\mathbb{P}_{n r e} \ln \left(e^{A^{-1} Y(\theta+\alpha) A} e^{g(\theta)} e^{-Y(\theta)}\right) \\
= & \mathbb{P}_{\text {nre }} \ln \left(e^{A^{-1}\left(Y(\theta+\alpha)+Y^{\prime}(\theta+\alpha)\right) A} e^{g(\theta)} e^{-\left(Y(\theta)+Y^{\prime}(\theta)\right)}\right) \\
& -\mathbb{P}_{\text {nre }} \ln \left(e^{A^{-1}\left(Y(\theta+\alpha)+Y^{\prime}(\theta+\alpha)\right) A} e^{g(\theta)} e^{-Y(\theta)}\right) \\
& +\mathbb{P}_{\text {nre }} \ln \left(e^{A^{-1}\left(Y(\theta+\alpha)+Y^{\prime}(\theta+\alpha)\right) A} e^{g(\theta)} e^{-Y(\theta)}\right) \\
& -\mathbb{P}_{\text {nre }} \ln \left(e^{A^{-1} Y(\theta+\alpha) A} e^{g(\theta)} e^{-Y(\theta)}\right)
\end{aligned}
$$

To make further computations, we need the fact that if $A, B, C$ are small $\operatorname{sl}(2, \mathbb{R})$ matrices, then there exists $D, E \in \operatorname{sl}(2, \mathbb{R})$ such that

$$
e^{A} e^{B} e^{C}=e^{D+E}
$$

and

$$
D=A+B+C
$$

where $E$ is a sum of terms of order at least 2 in $A, B, C$. Also, the famous Baker-Campbell-Hausdorff Formula shows that

$$
\ln \left(e^{X} e^{Y}\right)=X+Y+\frac{1}{2}[X, Y]+\frac{1}{12}([X,[X, Y]+[Y,[Y, X]])+\cdots
$$

where $[X, Y]=X Y-Y X$ denotes the Lie Bracket and $\cdots$ denotes the sum of higher order terms. 
Therefore, we can compute that

$$
\begin{aligned}
& \mathbb{P}_{\text {nre }} \ln \left(e^{A^{-1}\left(Y(\theta+\alpha)+Y^{\prime}(\theta+\alpha)\right) A} e^{g(\theta)} e^{-\left(Y(\theta)+Y^{\prime}(\theta)\right)}\right) \\
- & \mathbb{P}_{\text {nre }} \ln \left(e^{A^{-1}\left(Y(\theta+\alpha)+Y^{\prime}(\theta+\alpha)\right) A} e^{g(\theta)} e^{-Y(\theta)}\right) \\
= & \mathbb{P}_{n r e} \ln \left(e^{A^{-1}\left(Y(\theta+\alpha)+Y^{\prime}(\theta+\alpha)\right) A} e^{g(\theta)} e^{-Y(\theta)} e^{-Y^{\prime \prime}(\theta)}\right) \\
- & \mathbb{P}_{n r e} \ln \left(e^{A^{-1}\left(Y(\theta+\alpha)+Y^{\prime}(\theta+\alpha)\right) A} e^{g(\theta)} e^{-Y(\theta)}\right) \\
= & \mathbb{P}_{\text {nre }} \ln \left(e^{D+E} e^{-Y^{\prime \prime}(\theta)}\right)-\mathbb{P}_{n r e} \ln \left(e^{D+E}\right) \\
= & \mathbb{P}_{\text {nre }}\left(D+E-Y^{\prime \prime}+\frac{1}{2}\left[D+E,-Y^{\prime \prime}\right]+\cdots\right)-\mathbb{P}_{\text {nre }}(D+E) \\
= & \mathbb{P}_{\text {nre }}\left(-Y^{\prime \prime}+\frac{1}{2}\left[D+E,-Y^{\prime \prime}\right]+\cdots\right)
\end{aligned}
$$

where

$$
\begin{gathered}
Y^{\prime \prime}(\theta)=Y^{\prime}(\theta)+\mathcal{O}(Y(\theta)) Y^{\prime}(\theta), \\
D(\theta)=A^{-1}\left(Y(\theta+\alpha)+Y^{\prime}(\theta+\alpha)\right) A+g(\theta)-Y(\theta),
\end{gathered}
$$

and $E$ is a sum of terms of order at least 2 in $A^{-1}\left(Y(\theta+\alpha)+Y^{\prime}(\theta+\right.$ $\alpha)) A, g(\theta),-Y(\theta)$.

Similarly, we have

$$
\begin{aligned}
& \mathbb{P}_{\text {nre }} \ln \left(e^{A^{-1}\left(Y(\theta+\alpha)+Y^{\prime}(\theta+\alpha)\right) A} e^{g(\theta)} e^{-Y(\theta)}\right) \\
- & \mathbb{P}_{\text {nre }} \ln \left(e^{A^{-1} Y(\theta+\alpha) A} e^{g(\theta)} e^{-Y(\theta)}\right) \\
= & \mathbb{P}_{\text {nre }} \ln \left(e^{Y^{\prime \prime \prime}} e^{A^{-1} Y(\theta+\alpha) A} e^{g(\theta)} e^{-Y(\theta)}\right) \\
- & \mathbb{P}_{\text {nre }} \ln \left(e^{A^{-1} Y(\theta+\alpha) A} e^{g(\theta)} e^{-Y(\theta)}\right) \\
= & \mathbb{P}_{\text {nre }} \ln \left(e^{Y^{\prime \prime \prime}} e^{F+H}\right)-\mathbb{P}_{n r e} \ln \left(e^{F+H}\right) \\
= & \mathbb{P}_{\text {nre }}\left(Y^{\prime \prime \prime}+F+H+\frac{1}{2}\left[Y^{\prime \prime \prime}, F+H\right]+\cdots\right)-\mathbb{P}_{\text {nre }}(F+H) \\
= & \mathbb{P}_{\text {nre }}\left(Y^{\prime \prime \prime}+\frac{1}{2}\left[Y^{\prime \prime \prime}, F+H\right]+\cdots\right)
\end{aligned}
$$

where

$$
\begin{gathered}
Y^{\prime \prime \prime}(\theta+\alpha)=A^{-1} Y^{\prime}(\theta+\alpha) A+\mathcal{O}\left(A^{-1} Y(\theta+\alpha) A\right) \times A^{-1} Y^{\prime}(\theta+\alpha) A, \\
F(\theta)=A^{-1} Y(\theta+\alpha) A+g(\theta)-Y(\theta)
\end{gathered}
$$

and $H$ is a sum of terms of order at least 2 in $A^{-1}(Y(\theta+\alpha) A, g(\theta),-Y(\theta)$.

By the definition of Frechet differential, we only need to consider the linear terms of $\Psi\left(Y+Y^{\prime}, g\right)-\Psi(Y, g)$, thus we have

$$
\begin{aligned}
D_{Y} \Psi(Y, g)\left(Y^{\prime}\right) & =\mathbb{P}_{n r e}\left(A^{-1} Y^{\prime}(\theta+\alpha) A+\mathcal{O}\left(A^{-1} Y(\theta+\alpha) A\right) \times A^{-1} Y^{\prime}(\theta+\alpha) A\right. \\
& \left.+\frac{1}{2}\left[Y^{\prime \prime \prime}, F+H\right]+\cdots\right) \\
& +\mathbb{P}_{n r e}\left(-Y^{\prime}(\theta)-\mathcal{O}(Y(\theta)) Y^{\prime}(\theta)+\frac{1}{2}\left[F+H^{\prime},-Y^{\prime \prime}\right]+\cdots\right),
\end{aligned}
$$


where $H^{\prime}$ is a sum of terms of order at least 2 in $A^{-1}(Y(\theta+\alpha) A, g(\theta),-Y(\theta)$. Moreover, the first "..." denotes the sum of terms which have order at least 2 in $F+H$ but only order 1 in $Y^{\prime \prime \prime}$. The second "..." denotes the sum of terms which have order at least 2 in $F+H^{\prime}$ but only order 1 in $Y^{\prime \prime}$.

Let $Y=0$ and $g=0$, then all the Lie brackets vanish. So we immediately obtain

$$
\begin{aligned}
D_{Y} \Psi(0,0)\left(Y^{\prime}\right) & =\mathbb{P}_{n r e}\left(A^{-1} Y^{\prime}(\theta+\alpha) A\right)+\mathbb{P}_{n r e}\left(-Y^{\prime}(\theta)\right) \\
& =A^{-1} Y^{\prime}(\theta+\alpha) A-Y^{\prime}(\theta)
\end{aligned}
$$

Thus

$$
\begin{aligned}
\left\|D_{Y} \Psi(0,0)\left(Y^{\prime}\right)\right\| & \geqslant\left|A^{-1} Y^{\prime}(\theta+\alpha) A-Y^{\prime}(\theta)\right|_{r} \\
& \geqslant \eta\left|Y^{\prime}\right|_{r} .
\end{aligned}
$$

So we have

$$
\left\|\left(D_{Y} \Psi(0,0)\right)^{-1}\right\| \leqslant \eta^{-1} .
$$

For our purpose, we set $s=\epsilon, \delta=\epsilon^{\frac{1}{2}}$ and $\eta \geqslant 13\|A\|^{2} \epsilon^{\frac{1}{2}}$. Then we have

$$
2 \times \underset{\sup _{B_{s}(0)}}{ }\|\Psi(0, g)\| \times\left\|\left(D_{Y} \Psi(0,0)\right)^{-1}\right\| \leqslant 2 \times \epsilon \times \frac{1}{13\|A\|^{2}} \epsilon^{-\frac{1}{2}} \leqslant \epsilon^{\frac{1}{2}}=\delta,
$$

then (5.1) is fulfilled.

On the other hand, direct computation shows that

$$
\begin{aligned}
& D_{Y} \Psi(Y, g)\left(Y^{\prime}\right)-D_{Y} \Psi(0,0)\left(Y^{\prime}\right) \\
= & \mathbb{P}_{n r e}\left(\mathcal{O}\left(A^{-1} Y(\theta+\alpha) A\right) \times A^{-1} Y^{\prime}(\theta+\alpha) A+\frac{1}{2}\left[Y^{\prime \prime \prime}, F+H\right]+\cdots\right) \\
+ & \mathbb{P}_{n r e}\left(-\mathcal{O}(Y(\theta)) Y^{\prime}(\theta)+\frac{1}{2}\left[F+H^{\prime},-Y^{\prime \prime}\right]+\cdots\right) .
\end{aligned}
$$

Therefore, we have

$$
\begin{aligned}
\sup _{\overline{B_{s}(0)} \times \overline{B_{\delta}(0)}}\left\|D_{Y} \Psi(Y, g)\left(Y^{\prime}\right)-D_{Y} \Psi(0,0)\left(Y^{\prime}\right)\right\| & \leqslant 6\left(\|A\|^{2}|Y|_{r}+\|A\|^{2}|g|_{r}\right)\left|Y^{\prime}\right| \\
& \leqslant 6\left(\|A\|^{2} \delta+\|A\|^{2} s\right)\left|Y^{\prime}\right| \\
& \leqslant 6\left(\|A\|^{2} \epsilon^{\frac{1}{2}}+\|A\|^{2} \epsilon\right)\left|Y^{\prime}\right|
\end{aligned}
$$

which implies

$$
\sup _{\overline{B_{s}(0)} \times \overline{B_{\delta}(0)}}\left\|D_{Y} \Psi(0,0)-D_{Y} \Psi(Y, g)\right\| \leqslant 6\left(\|A\|^{2} \epsilon^{\frac{1}{2}}+\|A\|^{2} \epsilon\right) .
$$


Thus we have

$$
\begin{aligned}
& \sup _{\overline{B_{s}(0)} \times \overline{B_{\delta}(0)}}\left\|I d_{\mathcal{B}_{r}^{n r e}(\eta)}-\left(D_{Y} \Psi(0,0)\right)^{-1} \times D_{Y} \Psi(Y, g)\right\| \\
& \leqslant \sup _{\overline{B_{s}(0)} \times \overline{B_{\delta}(0)}}\left\|D_{Y} \Psi(0,0)-D_{Y} \Psi(Y, g)\right\| \times\left\|\left(D_{Y} \Psi(0,0)\right)^{-1}\right\| \\
& \leqslant 6\left(\|A\|^{2} \epsilon^{\frac{1}{2}}+\|A\|^{2} \epsilon\right) \times \frac{1}{13\|A\|^{2}} \epsilon^{-\frac{1}{2}} \\
& \leqslant \frac{1}{2}
\end{aligned}
$$

which satisfies (5.2). By Theorem 5.1, for $|g|_{r} \leqslant \epsilon$ and $\eta \geqslant 13\|A\|^{2} \epsilon^{\frac{1}{2}}$, there exists $|Y|_{r} \leqslant \epsilon^{\frac{1}{2}}$ such that $\Psi(Y, g)=0$, i.e.

$$
e^{A^{-1} Y(\theta+\alpha) A} e^{g(\theta)} e^{Y(\theta)}=e^{g^{r e}(\theta)},
$$

which is equivalent to

$$
e^{Y(\theta+\alpha)} A e^{g(\theta)} e^{Y(\theta)}=A e^{g^{r e}(\theta)}
$$

and it is easy to check $\left|g^{r e}(\theta)\right|_{r} \leqslant 2 \epsilon$. This finishes the proof of Lemma 3.1.

\section{AcKnowledgements}

C. Chavaudret was supported by the ANR "BEKAM" and the ANR "Dynamics and CR Geometry". J. You was partially supported by NSFC grant (11471155) and 973 projects of China (2014CB340701). Q. Zhou was partially supported by "Deng Feng Scholar Program B" of Nanjing University, Specially-appointed professor programe of Jiangsu province and NSFC grant (11671192).

\section{REFERENCES}

[1] S. Amor, Hölder continuity of the rotation number for the quasi-periodic cocycles in $S L(2, \mathbb{R})$. Commun. Math. Phys. 287 (2009), 565-588.

[2] A. Avila, The absolutely continuous spectrum of the almost Mathieu operator. Preprint.

[3] A. Avila, Global theory of one-frequency Schrödinger operators. Acta Math. 215 (2015), 1-54.

[4] A. Avila; S. Jitomirskaya, Almost localization and almost reducibility. J. Eur. Math. Soc. 12 (2010), 93-131.

[5] A. Avila; S. Jitomirskaya; C. Sadel, Complex one-frequency cocycles. J. Eur. Math. Soc. 16 (2013), 1915-1935.

[6] A. Avila; R. Krikorian, Monotonic cocycles. Invent. Math. 202 (2015), 271-331.

[7] A. Avila; M. Viana; A. Eskin, Continuity of Lyapunov exponents of random matrix products. In preparation.

[8] A. Avila; J. You; Q. Zhou, Sharp phase transitions for the almost Mathieu operator. Duke Math. DOI 10.1215/00127094-2017-0013. 
[9] L. Backes; A. W Brown; C. Butler, Continuity of Lyapunov exponents for cocycles with invariant holonomies. (2015) arXiv preprint arXiv:1507.08978.

[10] M. Berti; L. Biasco, Forced vibrations of wave equations with nonmonotone nonlinearities. Ann. I. H. Poincaré-AN. 23 (2006), 439-474

[11] C. Bocker-Neto; M. Viana, Continuity of Lyapunov Exponents for Random 2D Matrices. Ergodic Theory and Dynamical Systems (2016) 1-30 doi:10.1017/etds.2015.116

[12] J. Bochi, Discontinuity of the Lyapunov exponent for non-hyperbolic cocycles. Unpublished. (1999)

[13] J. Bochi, Genericity of zero Lyapunov exponents. Ergodic Theory and Dynamical Systems 22(6) (2002), 1667-1696.

[14] J. Bourgain, Positivity and continuity of the Lyapounov exponent for shifts on $T^{d}$ with arbitrary frequency vector and real analytic potential. J. Anal. Math. 96 (2005), 313-355.

[15] J. Bourgain; S. Jitomirskaya, Continuity of the Lyapunov exponent for quasiperiodic operators with analytic potential. J. Stat. Phys. 108 (2002), 1203-1218.

[16] J. Bourgain, Hölder regularity of integrated density of states for the almost Mathieu operator in a perturbative regime. Lett. Math. Phys. 51-2 (2000),83-118.

[17] C. Chavaudret, Strong almost reducibility for analytic and gevrey quasiperiodic cocycles. Bull. Soc. Math. France (2011)

[18] C. Chavaudret, Almost reducibility for finitely differentiable $S L(2, \mathbb{R})$ valued quasi-periodic cocycles. Nonlinearity 25(2012), 481-494.

[19] K.Deimling, Nonlinear functional analysis. Berlin-Heidelberg-NewYork: Springer-Verlag (1985)

[20] L. H. Eliasson, Floquet solutions for the 1-dimensional quasi-periodic Schrödinger equation. Commun. Math. Phys. 146 (1992), 447-482.

[21] A. Furman, On the multiplicative ergodic theorem for the uniquely ergodic systems. Ann. Inst. Henri Poincaré 33 (1997),797-815.

[22] M. Goldstein; W. Schlag, Hölder continuity of the integrated density of stats for quasi-periodic Schródinger equations and averages of shifts of subharmonic functions. Ann. Math. 154-1 (2001), 155-203.

[23] M. Goldstein; W. Schlag, Fine properties of the integrated density of states and a quantitative separation property of the Dirichlet eigenvalues. Geom. Funct. Anal. 18 (2008), 755-869.

[24] X. Hou; J. You, Almost reducibility and non-perturbative reducibility of quasiperiodic linear systems. Invent. Math. 190 (2012), 209-260.

[25] S. Jitomirskaya; I. Kachkovskiy, All couplings localization for quasiperiodic operators with Lipschitz monotone potentials. To appear in JEMS (2015)

[26] S. Jitomirskaya; D. A. Koslover; M.S. Schulteis, Continuity of the Lyapunov exponent for analytic quasiperiodic cocycles. Ergodic Theory and Dynamical Systems 29 (2009), 1881-1905. 
[27] S. Klein, Localization for quasi-periodic Schrödinger operators with multivariable Gevrey potential functions. J. Spec. Theory 4 (2014), 1-53

[28] O. Knill, The upper Lyapunov exponent of $S L(2, \mathbb{R})$ cocycles: Discontinuity and the problem of positivity. Lecture notes in Math. 1486 (1990), 86-97.

[29] M. Leguil; J. You; Z. Zhao; Q. Zhou, Asymptotics of spectral gaps of quasi-periodic Schrödinger operators. Preprint.

[30] J. Thouvenot, An example of discontinuity in the computation of the Lyapunov exponents. Proc. Stekolov Inst. Math. 216 (1997), 366-369.

[31] M. Viana, Lectures on Lyapunov Exponents. Cambridge Studies in Advanced Mathematics, Cambridge University Press (2014)

[32] Y. Wang; J. You, Examples of discontinuity of Lyapunov exponent in smooth quasi-periodic cocycles. Duke Math. 162 (2013), 2363-2412.

[33] Y. Wang; Z. Zhang, Uniform positivity and continuity of Lyapunov exponents for a class of $C^{2}$ quasi-periodic Schrödinger Cocycles. J. Func. Anal. 268 (2015), 2525-2585.

[34] E. Zehnder, Generalized implicit function theorems with applications to some small divisor problems:I. Comm. Pure Appl. Math. XXVIII (1975), 91-140.

Department of Mathematics, Nanjing University, Nanjing 210093, China

E-mail address: godcaiao@126.com

Laboratoire J.A. Dieudonné, Université de Nice-Sophia Antipolis (Parc Valrose), 06108 Nice Cedex 02, France

E-mail address: claire.chavaudret@unice.fr

Chern Institute of Mathematics and LPMC, Nankai University, Tianjin 300071, CHINA

E-mail address: jyou@nankai.edu.cn

Department of Mathematics, Nanjing University, Nanjing 210093, China

E-mail address: qizhou628@gmail.com, qizhou@nju.edu.cn 